\title{
Flavor anomalies from asymptotically safe gravity
}

\author{
Kamila Kowalska $^{\mathrm{a}}$, Enrico Maria Sessolo ${ }^{\mathrm{b}}$, Yasuhiro Yamamoto $^{\mathrm{c}}$ \\ National Centre for Nuclear Research, Pasteura 7, 02-093 Warsaw, Poland
}

Received: 16 July 2020 / Accepted: 20 March 2021 / Published online: 1 April 2021

(C) The Author(s) 2021

\begin{abstract}
We use the framework of asymptotically safe quantum gravity to derive predictions for scalar leptoquark solutions to the $b \rightarrow s$ and $b \rightarrow c$ flavor anomalies. The presence of an interactive UV fixed point in the system of gauge and Yukawa couplings imposes a set of boundary conditions at the Planck scale, which allows one to determine lowenergy values of the leptoquark Yukawa matrix elements. As a consequence, the allowed leptoquark mass range can be significantly narrowed down. We find that a consistent gravity-driven solution to the $b \rightarrow s$ anomalies predicts a leptoquark with the mass of 4-7 $\mathrm{TeV}$, entirely within the reach of a future hadron-hadron collider with $\sqrt{s}=100 \mathrm{TeV}$. Conversely, in the case of the $b \rightarrow c$ anomalies the asymptotically safe gravity framework predicts a leptoquark mass at the edge of the current LHC bounds. Complementary signatures appear in flavor observables, namely the (semi)leptonic decays of $B$ and $D$ mesons and kaons.
\end{abstract}

\section{Introduction}

Recent years have seen substantial development in the field of asymptotically safe quantum gravity [1-14]. An ambitious program has emerged around the fact that graviton fluctuations induce in the trans-Planckian regime universal contributions to the renormalization group (RG) running of matter couplings [15-30] that can be calculated via the functional renormalization group [31]. It has also been established that, given the matter content of a certain theory under study, at the energy scale where gravity is not negligible the renormalization group system of the gravity and matter couplings can develop a non-trivial (interactive) fixed point [27,32-35]. This feature has important consequences for our understanding of whether a given quantum field theory can be consid-

\footnotetext{
a e-mail: kamila.kowalska@ncbj.gov.pl (corresponding author)

b e-mail: enrico.sessolo@ncbj.gov.pl

c e-mail: yasuhiro.yamamoto@ncbj.gov.pl
}

ered fundamental. For example, in the standalone Standard Model (SM) the running of the hypercharge gauge coupling encounters eventually a Landau pole in the deep ultraviolet (UV), but the presence of gravitational interactions can tame its running by antiscreening graviton fluctuations, so that the gauge coupling remains finite $[27,32,33]$. Thus, the theory with a UV fixed point can be non-perturbatively renormalizable.

The important finding that quantum gravity and matter can feature interactive fixed points in the extreme trans-Planckian regime has opened the exciting possibility of deriving the values of the observable quantities of the SM (gauge, Yukawa and scalar couplings) from first principles [34-37]. The RG flow of the system, emerging from the UV fixed point, down to the electroweak symmetry breaking (EWSB) scale along the UV-safe trajectory, has led in fact to specific predictions (or postdictions in some cases) for the top Yukawa coupling [34] and the quartic coupling of the Higgs potential [36]. This framework was also used to predict the Lagrangian couplings of a few New Physics (NP) models extending the SM by an extra U(1) gauge symmetry and a scalar field with portal couplings [38-42].

In the spirit of the above-cited works, in this paper we try to investigate whether the embedding in an asymptoticallysafe gravity framework could improve the predictivity of particular NP models for which some experimental information exists, but it is still incomplete and/or not sufficient to draw a clear direction for future searches that should confirm unequivocally the existence of the NP itself and uncover its properties. A practical example we have in mind has to do with the so-called flavor anomalies in $b \rightarrow s$ [43-54] and $b \rightarrow c$ [55-64] transitions. These are a set of measurements, reported over the past several years by $\mathrm{LHCb}$ and other experimental collaborations, which suggest to high statistical significance a departure from the SM predictions of certain decay amplitudes involving a change of flavor of the participating fermions. NP solutions to the flavor anomalies are usually cast in terms of bounds on certain products of masses 
and couplings, but the current experimental information does not allow to pinpoint the particular mass scale at which the NP could be directly observed. An extra piece of theoretical information, provided for example by specific boundary conditions of the NP Yukawa couplings in the deep UV, could help to bridge this gap.

In this study we focus on the well known scalar leptoquark (LQ) solutions to the flavor anomalies (for a review, see, e.g., Ref. [65]). Scalar LQs provide a simple, single-field addition to the SM that, in the first approximation, does not need to be embedded with care into an additional UV completion but instead necessitates only of some basic assumptions on the scalar potential. On the other hand, LQs bring their own bag of complications to the fixed-point analysis, which we will discuss in detail in the following sections. First and foremost, they are not expected to be flavor-universal and there is in principle no knowledge of the alignment between the LQ and SM Yukawa matrices in flavor space. In practice, one can pick a particular basis for the fixed-point analysis and make sure that the basis remains well-defined along the entire RG flow. We will choose to work in the quark mass basis, in which the SM Yukawa matrices remain diagonal. As a direct consequence, we will treat the Cabibbo-Kobayashi-Maskawa (CKM) matrix parameters as renormalizable dimensionless couplings of the Lagrangian and add them to the fixed-point analysis as was done, e.g., in Ref. [37].

We will show that, if we require consistency with the neutral-current, $b \rightarrow s$ flavor anomalies, the information derived from the trans-Planckian fixed-point analysis leads to very specific predictions for the mass of the LQ, which should lie approximately in the 5-10 TeVrange. This places it outside of the foreseeable reach of the LHC, but well within the early reach of a $100 \mathrm{TeV}$ hadron machine according to the most conservative estimates. On the other hand, as the strength required for a NP contribution to the $b \rightarrow c$ anomalies is larger than in the neutral current case, our analysis confirms the well known fact that the charged-current anomalies point to new states just above the current sensitivity of the LHC. In this sense, one does not draw new indications from the UV completion, but we find it interesting per se that this kind of solutions can be made consistent with a theoretical embedding so far unexplored in this context.

The structure of the paper is the following. In Sect. 2 we give a brief overview of asymptotic safety in quantum gravity and we recall the mathematical setup used for the UV fixed-point analysis. In Sect. 3 we introduce the scalar LQ explanation for the $b \rightarrow s$ flavor anomalies. Subsections are dedicated to the full fixed-point analysis, a description of its solutions, and a summary of its low-scale predictions. In Sect. 4 we provide the full fixed-point analysis, description of solutions, and summary of low-scale predictions for the scalar LQ involved in the $b \rightarrow c$ case. We summarize our findings in Sect. 5. Some technical details of the LQ models and of the RG flow analyses are given in Appendix A and Appendix B.

\section{Asymptotic safety from quantum gravity}

In the presence of non-negligible gravitational interactions, a regime expected to be entered while approaching the Planck scale, the RG flow of matter couplings is modified [15-30,32, $33]$. For generic gauge $(g)$ and Yukawa $(y)$ couplings, such gravity-corrected beta functions are schematically given by

$\beta_{g}=\beta_{g}^{\mathrm{SM}+\mathrm{NP}}-g f_{g}$,

$\beta_{y}=\beta_{y}^{\mathrm{SM}+\mathrm{NP}}-y f_{y}$,

where $\beta_{x} \equiv d x / d \log Q$, and the first term on the right hand side in both equations denotes standard contributions to the $\mathrm{RG}$ running from the SM and NP. The effect of gravitational interactions, captured by the parameters $f_{g}$ and $f_{y}$, is universal in the sense that gravity distinguishes between different types of matter interactions (gauge, Yukawa, scalar quartic, etc.), while, being blind to the internal symmetries, does not explicitly depend on the corresponding couplings. Note that in Eqs. (1) and (2) we neglect possible quantum gravity effects proportional to higher powers in the matter couplings.

In the presence of asymptotically safe quantum gravity, the gravity-induced contributions $f_{g}$ and $f_{y}$ are determined by both the gravitational dynamics and the matter content of the coupled theory, whether this is the SM or its NP extension $[21,26,29,30,66]$. While the SM and NP contributions are well defined in a given NP framework, the theoretical status of the parameters $f_{g}$ and $f_{y}$ is far from being definitively settled.

It was shown in Ref. [24] that the leading quantum gravity contribution to the gauge coupling beta functions cannot be negative, irrespective of a chosen RG scheme. In particular, one can prove that $f_{g}=0$ as long as the RG scheme preserves a certain symmetry of the classical gauge-gravity Lagrangian, whereas strictly positive values are obtained as soon as one breaks this symmetry, irrespective of any other technical choices. Note, from Eq. (1), that a strictly positive $f_{g}$ is required to enforce asymptotic freedom in the gauge sector, and in this sense one is inclined to choose an RG scheme in which the leading non-universal coefficient is nonzero to be consistent with the low-energy phenomenology. Conversely, higher-order calculations would be required to determine the fate of theories with $f_{g}=0$. The existence of a non-trivial combined fixed point in a coupled system of gravity and matter has been confirmed in Ref. [29]. It was shown there that in such a setup gravity is asymptotically safe, while the gauge sector asymptotically free (see also Ref. [67] for an early review). 
The theoretical status of the leading-order gravity correction $f_{y}$ is less clear. Only a set of simplified models has been analyzed in the literature in this context [20,21,25,26], but no general results and definite conclusions regarding the sign of $f_{y}$ are available. Note, however, that quantum gravity is indispensable to generate a weakly coupled Yukawa fixed point $[68,69]$.

Additional unknowns adhere to the issue of how the gravity sector can carry the addition of matter, with large uncertainties that can stem from various sources. The first one is the choice of truncation of the theory space. In Einstein-Hilbert truncation only two operators in the scale-dependent effective action are retained, leading to the gravitational dynamics being governed exclusively by the Newton and cosmological constants [2]. Inclusion of higher order interactions enriches the theory by additional free parameters [5, 10,70-72]. Secondly, within a chosen truncation, the derivation of gravity contributions to the matter beta functions is cutoff-scheme dependent $[4,73]$ and various results can differ by up to 50 $60 \%$ [66].

For all these reasons, we will follow the approach of Refs. $[34,35,41,42]$ and treat $f_{g}$ and $f_{y}$ as free parameters whose specific values will define a particular set of boundary conditions for the SM and NP couplings at the Planck scale, obtained by following the RG flow of the coupling system from the UV fixed point. On the other hand, the requirement of matching the SM parameters onto their experimentally measured values imposes strong limitations on the allowed magnitude of $f_{g}$ and $f_{y}$, where the former is usually determined by the low-energy hypercharge coupling.

A fixed point of the system of Eqs. (1) and (2) is given by any set $\left\{g^{*}, y^{*}\right\}$, generically indicated with an asterisk, such that $\beta_{g}\left(g^{*}, y^{*}\right)=\beta_{y}\left(g^{*}, y^{*}\right)=0$. In order to determine the structure of the fixed point one needs to analyze the RG flow in its vicinity. The standard method is to linearize the $\mathrm{RG}$ equation system of the couplings, $\left\{\alpha_{i}\right\} \equiv\{g, y\}$, around the fixed point. One derives the stability matrix, $M$,

$M_{i j}=\partial \beta_{i} /\left.\partial \alpha_{j}\right|_{\left\{\alpha_{i}^{*}\right\}}$,

whose eigenvalues $\theta_{i}$, called critical (or scaling) exponents, characterize the power-law evolution of the couplings in the vicinity of $\left\{\alpha_{i}^{*}\right\}$.

If a critical exponent is negative the corresponding eigendirection is UV attractive and dubbed as relevant. All the RG trajectories along this direction will asymptotically reach the fixed point. A deviation of a relevant coupling from the fixed point introduces a free parameter in the theory and this freedom can be used to fine tune the coupling at some high scale to match an eventual measurement in the infrared (IR). Conversely, if an eigenvalue of $M$ is positive, the corresponding eigendirection is UV repulsive and commonly dubbed as irrelevant. In this case there exist only one trajectory the coupling's flow can follow in its run to the IR, thus providing potentially a clear prediction for its value at the low, experimentally interesting scale. All the trajectories which emanate from the UV stable fixed points correspond to theories that remain finite at high energies. Finally, $\theta_{i}=0$ introduces a marginal eigendirection. The RG flow along this direction is logarithmically slow and one needs to go beyond the linear approximation to decide whether a fixed point is attractive or repulsive.

\section{Flavor anomalies in $b \rightarrow s$ transitions}

We consider in this section the anomalies recorded in the last several years at LHCb [43-50], Belle [51,52], CMS [53], and ATLAS [54], involving substantial deviations from the SM in the measured values of the lepton-flavor violating ratios and angular distributions of the decays $B \rightarrow K^{(*)} \mu \mu$. Numerous global fits [74-93] have pointed to the likely emergence of NP in the effective operators

$\mathscr{O}_{9}^{\mu(\prime)}=\frac{\alpha_{\mathrm{em}}}{4 \pi}\left(\bar{s} \gamma_{\rho} P_{L(R)} b\right)\left(\bar{\mu} \gamma^{\rho} \mu\right)$,
$\mathscr{O}_{10}^{\mu(\prime)}=\frac{\alpha_{\mathrm{em}}}{4 \pi}\left(\bar{s} \gamma_{\rho} P_{L(R)} b\right)\left(\bar{\mu} \gamma^{\rho} \gamma^{5} \mu\right)$,

the statistical significance of which exceeds, according to some analyses, the $5 \sigma$ level.

Among the NP scenarios well suited to induce the required deviation in the Wilson coefficient $C_{9}^{\mu},{ }^{1} \mathrm{LQs}$ are particularly appealing for their simplicity. For example, the anomalies could be explained by the tree-level exchange of a single component of the scalar LQ $S_{3}$ [65,94-101], which is a triplet of $\mathrm{SU}(2)_{L}$. However, as is generally the case when matching renormalizable NP models to the flavor constraints expressed in terms of operators of dimension higher than 4 , even a relatively precise measurement of the corresponding Wilson coefficient does not suffice to pinpoint the scale and interaction strength of the LQ independently, as the constraints are expressed in terms of a ratio coupling/mass. Thus, in order to make specific predictions, one has to introduce some assumptions on the nature of the UV completion. We show here that asymptotically safe gravity provides a predictive high-energy framework for the $S_{3}$ LQ in the context of the $b \rightarrow s$ anomalies.

We remind the reader that a solution to the flavor anomalies implies (we constrain ourselves for simplicity to the single dimension $C_{9}^{\mu}=-C_{10}^{\mu}$ )

$C_{9}^{\mu}=-C_{10}^{\mu} \in(-0.7,-0.3)$

at the $2 \sigma$ level [91]. The corresponding operator can be generated by the $\mathrm{LQ} S_{3}$, whose $\mathrm{SU}(3)_{c} \times \mathrm{SU}(2)_{L} \times \mathrm{U}(1)_{Y}$ quantum

\footnotetext{
${ }^{1}$ A negative deviation from the SM expectation of $C_{9}^{\mu}$ alone is sufficient to explain the data, but other operators can be nonzero too, $\mathrm{cf}$. the global fits cited above.
} 
numbers are $(\overline{\mathbf{3}}, \mathbf{3}, 1 / 3)$. We can write down the interaction Lagrangian in terms of left-chiral two-component spinors in the mass basis (we use the Weyl notation throughout this work),

$$
\begin{aligned}
\mathscr{L} \supset & \hat{Y}_{i j}^{L}\left(-\phi_{1 / 3} d_{L, i} v_{L, j}-\sqrt{2} \phi_{4 / 3} d_{L, i} e_{L, j}\right) \\
& +\widetilde{Y}_{i j}^{L}\left(\sqrt{2} \phi_{-2 / 3} u_{L, i} \nu_{L, j}-\phi_{1 / 3} u_{L, i} e_{L, j}\right)+\text { H.c. }
\end{aligned}
$$

where a sum over repeated SM generation indices is intended, numbers in subscripts indicate the scalar fields' electric charge, and the up- and down-type couplings are related to each other by the CKM matrix $V$ as $\widetilde{Y}_{i j}=V_{i k}^{*} \hat{Y}_{k j}$. Note that we do not address in this work the physics of neutrinos, which we assume form a separate system not affecting the fixedpoint analysis of states that are heavier by several orders of magnitude. We thus constrain ourselves to a SM-like framework, in which the charged lepton generations do not mix with one another. Additional details on the $S_{3}$ Lagrangian and the complete list of adopted assumptions are given in Appendix A.

By matching to the $\mathscr{O}_{9}^{\mu}$ operator via the $t$-channel exchange of $\phi_{4 / 3}$ one gets

$C_{9}^{\mu}=-C_{10}^{\mu}=\frac{\pi v_{h}^{2}}{V_{33} V_{32}^{*} \alpha_{\mathrm{em}}} \frac{\hat{Y}_{32}^{L} \hat{Y}_{22}^{L *}}{m_{S_{3}}^{2}}$,

where $v_{h}$ is the Higgs vev, $\alpha_{\mathrm{em}}$ is the fine structure constant, and we have attributed a common mass $m_{S_{3}}$ to the triplet's states.

Equation (5) leads to the $2 \sigma$ bound

$0.4 \times 10^{-3}\left(\frac{m_{S_{3}}}{\mathrm{TeV}}\right)^{2} \leq \hat{Y}_{32}^{L} \hat{Y}_{22}^{L *} \leq 1.1 \times 10^{-3}\left(\frac{m_{S_{3}}}{\mathrm{TeV}}\right)^{2}$,

which, as was explained above, does not provide an independent determination of the LQ mass and coupling. On the other hand, if a specific value of the Yukawa couplings were to emerge from the UV completion, Eq. (8) would provide a clear indication of the LQ mass scale, subject only to the experimental precision of the flavor measurements. In the next subsection we derive the value of the LQ Yukawa coupling from the fixed-point analysis of the system in minimally coupled quantum gravity in the trans-Planckian regime.

\subsection{Fixed-point analysis}

Since we seek to connect the high-scale boundary conditions with observable quantities at the low scale, we derive the renormalization group equations (RGEs) in the quark mass basis. Besides, since we use specifically the measurements of $b \rightarrow s$ anomalies to constrain the NP system at the low scale, we choose to work in a down-origin basis for the $S_{3}$
Yukawa matrices. A more detailed discussion of our choice of basis can be found in Appendix A.

As the Yukawa matrices of the SM and those of the LQ system do not necessarily commute, we are left with diagonal Yukawa entries for the SM and arbitrary textures for the down-type $\hat{Y}_{i j}^{L}$ matrices. However, since we neglect the neutrino mass, we can always choose a charged-lepton Yukawa matrix diagonal in flavor space and thus we do not generate inter-column, charged lepton-flavor violating elements via RG flow. The CKM matrix elements are subject to $R G$ running like the SM and NP Yukawa couplings. Above the Planck scale, the system is coupled to the quantum fluctuations of the graviton, which introduce the $f_{g}$ and $f_{y}$ terms in the RGEs and give rise to the possible emergence of UV (Gaussian and interactive) fixed points.

In the present analysis we focus only on the parameters affecting the phenomenological $b \rightarrow s$ constraints. This means that we work effectively in a 2-family (second + third) approximation in which the CKM matrix is parametrized by one single rotation angle. Additionally, we do not include in the analysis the Yukawa couplings of the quarks of the first two generations, as their impact on the running of other parameters is negligible. One should expect to be able to set these negligible parameters along a relevant direction of a Gaussian fixed point in the trans-Planckian UV [37].

The minimal system of couplings consists of 8 independent parameters,

$g_{Y}, g_{2}, g_{3}, y_{t}, y_{b}, \hat{Y}_{22}^{L}, \hat{Y}_{32}^{L}, V_{33}$,

where, respectively, $g_{Y}, g_{2}$ and $g_{3}$ are the gauge couplings of $\mathrm{U}(1)_{Y}, \mathrm{SU}(2)_{L}$ and $\mathrm{SU}(3)_{c}$, and $y_{t}$ and $y_{b}$ denote the Yukawa couplings of top and bottom quarks. Note that the Yukawa coupling $\hat{Y}_{12}^{L}$ does not enter the fixed-point analysis in our approximation. We do make sure, however, that if it is assumed to be zero at the Planck scale, it does not get renormalized at the low scale into values in tension with the experimental bounds. Finally, we point out that we limit our analysis to real couplings only. The relevant RGEs for the $S_{3}$ plus $\mathrm{SM}$ system coupled to gravity are given in Appendix A. ${ }^{2}$

Let us explore the structure of the fixed points for the system given by Eq. (9). The non-abelian gauge couplings develop non-interactive UV fixed points, indicated hence-

\footnotetext{
${ }^{2}$ It is worth pointing out that we do not incorporate the parameters of the scalar potential in the fixed-point analysis. They do not enter at one loop in the RGEs of the gauge-Yukawa system, for which we derive the phenomenological predictions related to the flavor anomalies, see Appendix A. Moreover, we have checked numerically that the predictions for the NP Yukawa couplings change minimally under the addition of perturbative 2-loop contributions to the beta functions. This variation is negligible with respect to the experimental uncertainty on the Wilson coefficients and does not affect the leptoquark mass determination.
} 
forth with an asterisk,

$g_{3}^{*}=0, \quad g_{2}^{*}=0$,

therefore they will correspond to the relevant directions in the coupling space. The trans-Planckian running of $g_{Y}$, on the other hand, is tamed by the graviton fluctuations, which lead to the generation of an interactive fixed point. As discussed in Sect. 2, matching the irrelevant $g_{Y}$ onto its phenomenological value in the IR allows one to unambiguously fix the parameter $f_{g}$,

$g_{Y}^{*}=4 \pi \sqrt{\frac{6 f_{g}}{43}}$.

One obtains $f_{g}=0.01$ and $g_{Y}^{*}=0.48$.

The second quantum gravity parameter, $f_{y}$, can be fixed if one of the SM Yukawa couplings presents a UV interactive fixed point [35], as in that case it is used to match the flow to the IR along the irrelevant direction onto the value of the corresponding quark mass. At the same time, the CKM matrix element $V_{33}$ must be set to zero at the fixed point to span a relevant direction [37]. The following combinations of the fixed-point values in the SM Yukawa sector are then possible:

$$
\begin{aligned}
& \mathrm{FP}_{1}: y_{t}^{*} \neq 0, \quad y_{b}^{*}=0, \quad V_{33}^{*}=0, \\
& \mathrm{FP}_{2}: y_{t}^{*}=0, \quad y_{b}^{*} \neq 0, \quad V_{33}^{*}=0, \\
& \mathrm{FP}_{3}: y_{t}^{*} \neq 0, \quad y_{b}^{*} \neq 0, \quad V_{33}^{*}=0 .
\end{aligned}
$$

Finally, three different sets of fixed-point values of the LQ Yukawa matrix entries can be obtained, allowing one of the two elements $\hat{Y}_{22}^{L}, \hat{Y}_{32}^{L}$ to be interactive, or none:

$$
\begin{aligned}
& \mathrm{FP}_{a}: \hat{Y}_{22}^{L *} \neq 0, \hat{Y}_{32}^{L *}=0, \\
& \mathrm{FP}_{b}: \hat{Y}_{22}^{L *}=0, \hat{Y}_{32}^{L *} \neq 0, \\
& \mathrm{FP}_{c}: \hat{Y}_{22}^{L *}=0, \hat{Y}_{32}^{L *}=0 .
\end{aligned}
$$

Note that a solution with both non-zero elements of the matrix $\hat{Y}_{i j}^{L}$ is not compatible with a relevant fixed point for $V_{33}$. The list of fixed points of phenomenological interest is summarized in Table 1 . The values assumed by $f_{y}$ at the various fixed points are also presented in Table 1.

The SM couplings correspond directly to eigendirections of the stability matrix. The relevant couplings are $g_{3}, g_{2}$, and the one among the top and bottom Yukawa couplings whose fixed-point value is zero, denoted with $y_{0}$ in what follows. The deviation of the relevant couplings from their UV fixed point at some high scale $\Lambda$ introduces several free parameters characterizing UV-safe trajectories running out of the fixed point:

$\delta g_{2,3}(\Lambda)=g_{2,3}^{*}-g_{2,3}(\Lambda), \quad \delta y_{0}=y_{0}^{*}-y_{0}(\Lambda)$.

Conversely, the irrelevant couplings $g_{Y}$ and $y_{\emptyset}$ (the SM Yukawa coupling(s) whose fixed-point value is nonzero) are expected to be entirely determined by their fixed-point value and thus constitute predictions of the theory. The behavior of the NP Yukawa couplings in the vicinity of the fixed point depends on which of the scenarios introduced in Eq. (13) is considered and will be discussed individually in the following paragraphs.

A word of caution is in order, though. The character of a coupling as a relevant parameter does not need to persist along its entire RG flow from the UV to the IR, as it may be affected by the existence of other fixed points in the system. In this regard, we anticipate here that all of the considered solutions found for the $\mathrm{SM}+S_{3}$ leptoquark system eventually cross over in their trans-Planckian flow to the basin of attraction of a fixed point characterized by IR-attractive $V_{33}^{*}=1$, $y_{t}^{*} \neq 0, y_{b}^{*}=0, \hat{Y}_{22}^{L *} \neq 0$ and $\hat{Y}_{32}^{L *}=0$. Explicitly,

$$
y_{t}^{*}=\frac{4 \pi}{3} \sqrt{\frac{17 f_{g}+86 f_{y}}{43}}, \quad \hat{Y}_{22}^{L *}=4 \pi \sqrt{\frac{10 f_{g}+86 f_{y}}{301}} .
$$

We dub this trans-Planckian IR fixed point as $\mathrm{FP}_{\mathrm{IR}}$. Its origin can be qualitatively understood by inspection of the RGEs presented at the end of Appendix A. Equations (A.17) and (A.19) imply that, because of the negative contributions induced by the hypercharge coupling, both $y_{t}$ and $\hat{Y}_{22}^{L}$ can eventually become substantial in their flow to the IR, independently of their starting point in the UV. The consequence of this growth is that the contribution proportional to $g_{Y}^{2}$ will be counterbalanced by $y_{t}^{2}$ in Eq. (A.17) and by $\left(\hat{Y}_{22}^{L}\right)^{2}$ in Eq. (A.19) (with some correction proportional to $y_{t} \hat{Y}_{32}^{L}$ that kicks in when $V_{33}^{2} \approx V_{32}^{2}$ ). This effect is dominated by the size of $g_{Y}^{*}$ and hence by the gravitational coupling $f_{g}$, and it is much less sensitive to the parameter $f_{y}$. As we shall see in the next paragraphs, in most of the cases of phenomenological interest the presence of $\mathrm{FP}_{\mathrm{IR}}$ along the RGE flow effectively washes out much of the freedom associated with relevant directions in the Yukawa-coupling theory space.

Scenarios of type $\mathrm{FP}_{a}$ For a fixed point of the type $\mathrm{FP}_{1 a}$ or $\mathrm{FP}_{3 a}$, the coupling $\hat{Y}_{22}^{L}$ is irrelevant, while the system $\left(V_{33}, \hat{Y}_{32}^{L}\right)$ spans a 2-dimensional submanifold in the coupling space, on which both couplings are relevant but do not correspond to eigendirections of the stability matrix.

The phase diagram of $\hat{Y}_{32}^{L}$ vs. $V_{33}$ in the vicinity of the fixed point is shown in Fig. 1a, with all the remaining couplings fixed at their fixed-point value. The fixed point is marked as a red dot, and the arrows indicate the RGE flow of the system towards the UV. For the trajectories shown in red, the fixed point can be reached from any direction, confirming that both couplings are indeed relevant. ${ }^{3}$ However, for any fixed deviation $\delta V_{33}$, there exists an upper bound on the allowed

\footnotetext{
${ }^{3}$ Note in Fig. 1a, that the streamlines flow with different speed along the $V_{33}$ and $\hat{Y}_{32}^{L}$ directions, giving the impression of entering the fixed point in the UV along one and the same line.
} 
Table 1 Fixed-point values and the corresponding $f_{y}$ of the $\mathrm{SM}+S_{3}$ Yukawa coupling system invoked for an explanation of the $b \rightarrow s$ anomalies. The low-energy prediction for the NP sector is shown in the last column on the right. The symbol † indicates those scenarios in which the predicted top mass exceeds the experimental value

\begin{tabular}{lllllll}
\hline & $f_{y}$ & $y_{t}^{*}$ & $y_{b}^{*}$ & $\hat{Y}_{22}^{L *}$ & $\hat{Y}_{32}^{L *}$ & \multicolumn{1}{c}{ Prediction } \\
\hline $\mathrm{FP}_{1 a}$ & 0.0014 & $4 \pi \sqrt{\frac{208 f_{g}+946 f_{y}}{5289}}$ & 0 & $4 \pi \sqrt{\frac{146 f_{g}+1376 f_{y}}{5289}}$ & 0 & $\hat{Y}_{22}^{L} \hat{Y}_{32}^{L}\left(m_{S_{3}}\right)<0$ \\
$\mathrm{FP}_{1 b}$ & 0.0088 & $4 \pi \sqrt{\frac{17 f_{g}+86 f_{y}}{387}}$ & 0 & 0 & $4 \pi \sqrt{\frac{10 f_{g}+86 f_{y}}{301}}$ \\
$\mathrm{FP}_{1 c}$ & 0.0024 & $4 \pi \sqrt{\frac{17 f_{g}+86 f_{y}}{387}}$ & 0 & 0 & $m_{S_{3}} \in[7,11] \mathrm{TeV} \dagger$ \\
$\mathrm{FP}_{2 a}$ & -0.0006 & 0 & $4 \pi \sqrt{\frac{5 f_{g}+86 f_{y}}{387}}$ & $4 \pi \sqrt{\frac{10 f_{g}+86 f_{y}}{301}}$ & 0 & $m_{S_{3}} \in[7,11] \mathrm{TeV} \dagger$ \\
$\mathrm{FP}_{2 b}$ & -0.0004 & 0 & $4 \pi \sqrt{\frac{40 f_{g}+946 f_{y}}{5289}}$ & 0 & $4 \pi \sqrt{\frac{170 f_{g}+1376 f_{y}}{5289}}$ & $\hat{Y}_{22}^{L} \hat{Y}_{32}^{L}\left(m_{S_{3}}\right)<0$ \\
$\mathrm{FP}_{2 c}$ & -0.0001 & 0 & $4 \pi \sqrt{\frac{5 f_{g}+86 f_{y}}{387}}$ & 0 & $m_{S_{3}} \in[4,7] \mathrm{TeV}$ \\
$\mathrm{FP}_{3 a}$ & 0.0014 & $4 \pi \sqrt{\frac{484 f_{g}+430 f_{y}}{8643}}$ & $4 \pi \sqrt{\frac{-211 f_{g}+1634 f_{y}}{8643}}$ & $4 \pi \sqrt{\frac{218 f_{g}+2408 f_{y}}{8643}}$ & 0 & $\hat{Y}_{22}^{L} \hat{Y}_{32}^{L}\left(m_{S_{3}}\right)<0$ \\
$\mathrm{FP}_{3 b}$ & 0.0087 & $4 \pi \sqrt{\frac{617 f_{g}+1634 f_{y}}{8643}}$ & $4 \pi \sqrt{\frac{-356 f_{g}+430 f_{y}}{8643}}$ & 0 & $4 \pi \sqrt{\frac{338 f_{g}+2408 f_{y}}{8643}}$ & $m_{S_{3}} \in[7,11] \mathrm{TeV}{ }^{\dagger}$ \\
$\mathrm{FP}_{3 c}$ & 0.0023 & $4 \pi \sqrt{\frac{41 f_{g}+86 f_{y}}{645}}$ & $4 \pi \sqrt{\frac{-19 f_{g}+86 f_{y}}{645}}$ & 0 & 0 & $m_{S_{3}} \in[7,11] \mathrm{TeV} \dagger$ \\
\hline
\end{tabular}

Fig. 1 a Phase diagram in the plane $\left(V_{33}, \hat{Y}_{32}^{L}\right)$ in the vicinity of the UV fixed point $\mathrm{FP}_{1 a}$, indicated here as a red dot. All the remaining couplings are set to their fixed-point values. The RG flow directions point towards the UV. Only the RG trajectories marked in red allow one to reach the UV fixed point. b Phase diagram in the plane $\left(V_{33}, \hat{Y}_{22}^{L}\right)$ in the vicinity of the $\mathrm{UV}$ fixed point $\mathrm{FP}_{1 b}$

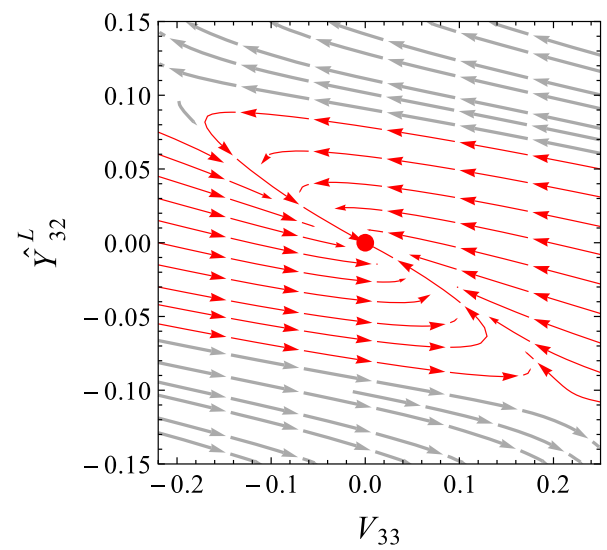

(a) $\mathrm{FP}_{1 a}$

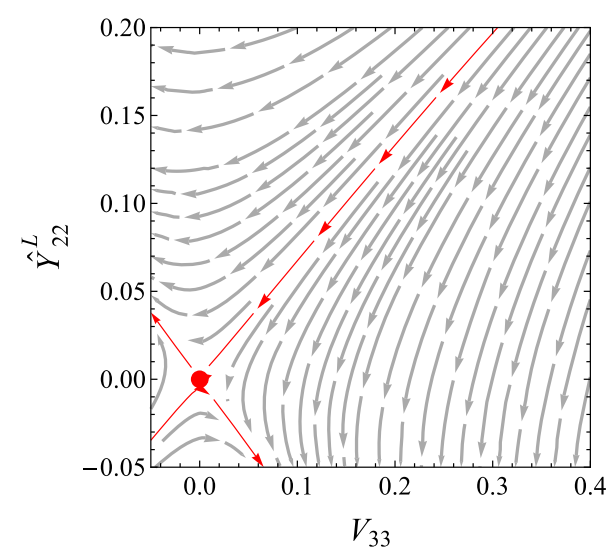

(b) $\mathrm{FP}_{1 b}$ size of the corresponding $\delta \hat{Y}_{32}^{L}$, and viceversa, which is due to the nontrivial beta function of the element $V_{33}$. This fact bears the important consequence that $\delta \hat{Y}_{32}^{L}$ never reaches a value large enough to guarantee that $\hat{Y}_{32}^{L}$ remain positive along the full length of its flow to the IR. More specifically, close to the relevant fixed point the running of $\hat{Y}_{32}^{L}$ is dominated by $y_{t}$, which introduces a positive contribution to the beta function [see Eq. (A.20) in Appendix A],

$\frac{d \hat{Y}_{32}^{L}}{d t} \simeq \frac{1}{16 \pi^{2}} \hat{Y}_{22}^{L} y_{t}^{2} V_{33} \sqrt{1-V_{33}^{2}}$.

Inevitably, in scenarios $\mathrm{FP}_{1 a}, \mathrm{FP}_{3 a}$ one obtains $\hat{Y}_{22}^{L} \hat{Y}_{32}^{L}<0$ at the low scale, in contradiction with the phenomenological requirement, cf. Eqs. (5), (7).

The situation is very similar for fixed point $\mathrm{FP}_{2 a}$, although in this case $\hat{Y}_{32}^{L}$ corresponds to an irrelevant direction. Once more, due to Eq. (16), the low-scale prediction is phenomenologically disfavored.

Scenarios of type $F P_{b}$ In this case both LQ Yukawa couplings become irrelevant directions. While $\hat{Y}_{32}^{L}$ corresponds to an eigenvector of the stability matrix, the flow of $\hat{Y}_{22}^{L}$ close to the fixed point is entirely dictated by the UV hypercritical surface relating it with the relevant CKM matrix element $V_{33}: \hat{Y}_{22}^{L}(t) \equiv \mathscr{F}\left(V_{33}(t)\right)$. The corresponding phase diagram is shown in Fig. 1b. Since in this case the main contribution to the $\hat{Y}_{22}^{L}$ beta function is negative [see Eq. (A.19) in Appendix $\mathrm{A}]$,

$\frac{d \hat{Y}_{22}^{L}}{d t} \simeq-\frac{2}{16 \pi^{2}} \hat{Y}_{32}^{L} y_{t}^{2} V_{33} \sqrt{1-V_{33}^{2}}$,

the product $\hat{Y}_{22}^{L} \hat{Y}_{32}^{L}$ is positive at the low scale, as required by global fits to the Wilson coefficients, $C_{9}^{\mu}=-C_{10}^{\mu}<0$. 
Fig. 2 RG flow of the gauge and Yukawa couplings from the trans-Planckian energies down to the EWSB scale for scenarios featuring the fixed point a $\mathrm{FP}_{1 b}$, and $\mathbf{b} \mathrm{FP}_{2 b}$. The sub-Planckian flow is depicted in the inset panels

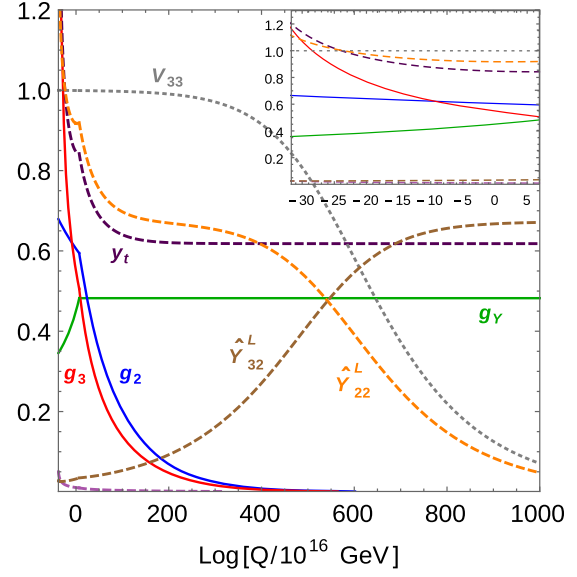

(a) $\mathrm{FP}_{1 b}$

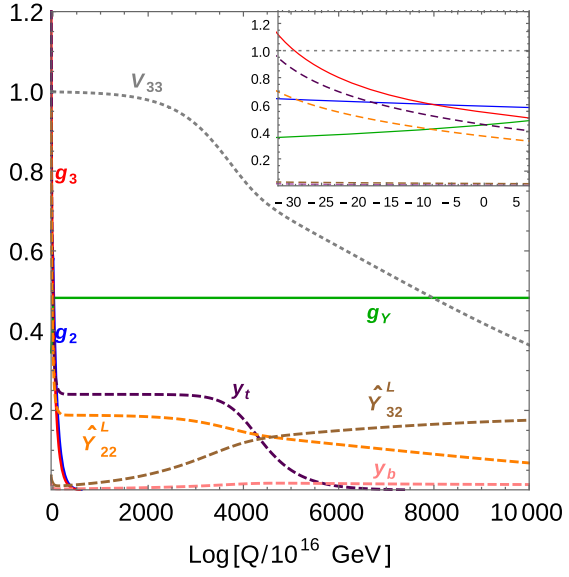

(b) $\mathrm{FP}_{2 b}$
The phenomenological predictions, obtained by following the RG flow of the examined coupling system from the UV fixed point towards the IR, differ somewhat for $\mathrm{FP}_{1 b}, \mathrm{FP}_{2 b}$, and $\mathrm{FP}_{3 b}$. In all three cases the requirement to fit the measured value of the hypercharge coupling, which we assume to be $g_{Y}\left(M_{t}\right)=0.3583$ [102], fixes $f_{g}=0.01$. Case $\mathrm{FP}_{1 b}$ features irrelevant nonzero top Yukawa coupling at the fixed point. But whether $y_{b}^{*}=0$ corresponds instead to a relevant or irrelevant direction - a key question from the point of view of the phenomenological viability of this scenario - hinges on the precise value of $f_{y}$. For small $f_{y}, y_{b}^{*}=0$ is irrelevant. Thus, we choose a minimal $f_{y}$ for which $y_{b}$ becomes relevant: $f_{y}=0.0088$. One then obtains the following low-energy predictions for the SM and LQ Yukawa couplings at $Q=$ $m_{S_{3}}=9 \mathrm{TeV}$,

$y_{t}\left(m_{S_{3}}\right)=1.07, \quad y_{b}\left(m_{S_{3}}\right)=0.01$,

$\hat{Y}_{22}^{L}\left(m_{S_{3}}\right)=1.04, \quad \hat{Y}_{32}^{L}\left(m_{S_{3}}\right)=0.05$.

The top mass is by around $20 \%$ too large with respect to the SM predictions - a price to pay for fitting correctly the bottom mass in scenario $\mathrm{FP}_{1 b}$.

The trans-Planckian flow of the parameters of the system is presented in Fig. 2a. Their IR behavior is determined by the crossover towards the basin of attraction of fixed point $\mathrm{FP}_{\mathrm{IR}}$, which happens around $\log Q \approx 300$ in Fig. 2a. $\mathrm{FP}_{\mathrm{IR}}$ is characterized by IR-attractive $V_{33}^{*}=1, y_{t}^{*} \approx 0.62, y_{b}^{*}=0$, and $\hat{Y}_{22}^{L *} \approx 0.67$, cf. Eq. (15). Since in this case $\hat{Y}_{32}^{L}$ is large and positive in the deep UV, it remains positive in its flow towards the IR while asymptotically approaching zero from above.

Given the fixed values of $f_{g}$ and $f_{y}$, the EWSB values of $\hat{Y}_{22}^{L}$, and $\hat{Y}_{32}^{L}$ are unambiguously predicted by the RG flow towards the IR after gravity decouples (see inset panel in Fig. 2a). Moreover, since both LQ couplings run slowly over several orders of magnitudes in scale, one can precisely determine the range of LQ masses for which the analyzed scenario is consistent with the explanation of $b \rightarrow s$ anomalies. It corresponds to $m_{S_{3}}=7-11 \mathrm{TeV}$.

Fixed point $\mathrm{FP}_{2 b}$ features a different behavior. The parameter $f_{y}$ is precisely determined by fitting $y_{b}$ to its IR value giving: $f_{y}=-0.0004$. At $Q=m_{S_{3}}=5 \mathrm{TeV}$ we obtain

$$
y_{t}\left(m_{S_{3}}\right)=0.83, y_{b}\left(m_{S_{3}}\right)=0.01 \text {, }
$$

$\hat{Y}_{22}^{L}\left(m_{S_{3}}\right)=0.63, \hat{Y}_{32}^{L}\left(m_{S_{3}}\right)=0.03$.

Note that the top mass can be matched onto the SM with a high degree of accuracy. The corresponding range of LQ mass reads $m_{S_{3}}=4-7 \mathrm{TeV}$. The trans-Planckian flow of the parameters of the system is presented in Fig. 2b. At the energies $Q \approx \exp (4000) \approx 10^{1700} \mathrm{GeV}$ one observes the crossover of the top Yukawa coupling from the basin of attraction of the $\mathrm{UV}$ fixed point $\mathrm{FP}_{2 b}$ to the basin of attraction of its IR fixed point $\mathrm{FP}_{\mathrm{IR}}$ with $y_{t}^{*} \approx 0.24$. It results in a characteristic "plateau" in the running of $y_{t}$ and allows for a good fit to the top mass once gravitational interactions decouple.

Finally, $\mathrm{FP}_{3 b}$ behaves quite similarly to $\mathrm{FP}_{1 b}$. While $f_{y}$ is fixed by the bottom mass, the predicted mass of the top quark at low-energies is once more a little too large. At $Q=$ $m_{S_{3}}=9 \mathrm{TeV}$ we obtain

$$
\begin{aligned}
y_{t}\left(m_{S_{3}}\right) & =1.07, \quad y_{b}\left(m_{S_{3}}\right)=0.013, \\
\hat{Y}_{22}^{L}\left(m_{S_{3}}\right) & =1.04, \quad \hat{Y}_{32}^{L}\left(m_{S_{3}}\right)=0.05 .
\end{aligned}
$$

Note that there is a difference between cases $\mathrm{FP}_{1 b}, \mathrm{FP}_{3 b}$, where $y_{t}$ is irrelevant with $f_{y}=0.0088$, and $\mathrm{FP}_{2 b}$, where it is relevant with $f_{y}=-0.00044$. The size of $f_{y}$ determines the size of the top Yukawa coupling at $\mathrm{FP}_{\mathrm{IR}}$, which reads 0.62 and 0.24 , respectively. In the latter case, $\left|f_{y}\right| \ll f_{g}$, similarly to one of the SM cases presented in Ref. [37], and this leads to a good top mass determination.

Scenarios of type $F P_{c}$ Fixed points of type $\mathrm{FP}_{c}$ are characterized by two relevant directions for the Gaussian $\hat{Y}_{22}^{L *}=0$ and $\hat{Y}_{32}^{L *}=0$. However, predictivity is restored in these cases by virtue of the flow of the CKM matrix element $V_{33}$. Matching 
Fig. 3 RG flow of the gauge and Yukawa couplings from the trans-Planckian energies down to the EWSB scale for scenarios featuring the fixed point a $\mathrm{FP}_{1 c}$, and $\mathbf{b} \mathrm{FP}_{2 c}$. The sub-Planckian flow is depicted in the inset panels

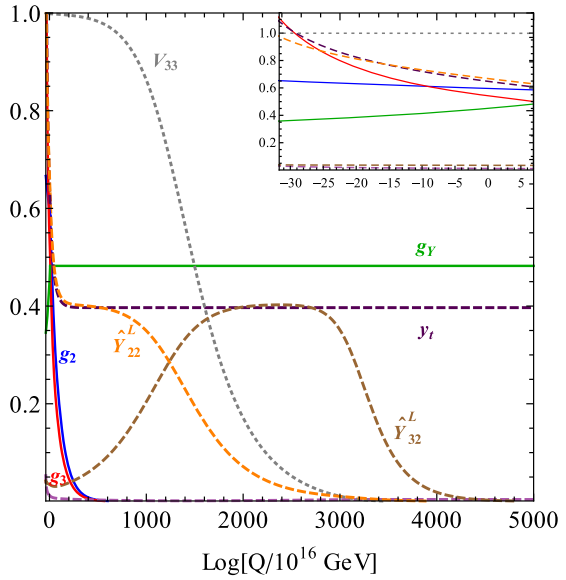

(a) $\mathrm{FP}_{1 c}$

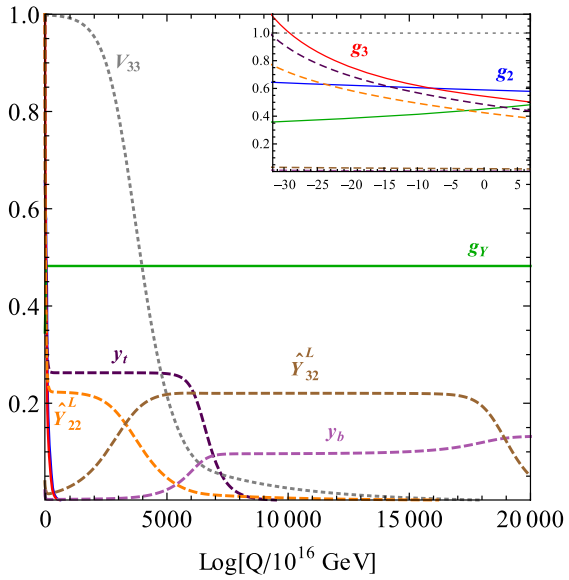

(b) $\mathrm{FP}_{2 c}$ it to its low-energy experimental determination once again drives the system, first to the basin of attraction of fixed points reminiscent of type $\mathrm{FP}_{b}$, and subsequently to the basin of attraction of FP IR. The latter determines the values of $\hat{Y}_{22}^{L}$ and $\hat{Y}_{32}^{L}$ at the Planck scale. The low-scale predictions for $\mathrm{FP}_{1 c}, \mathrm{FP}_{2 c}$, and $\mathrm{FP}_{3 c}$ resemble closely the predictions for $\mathrm{FP}_{1 b}, \mathrm{FP}_{2 b}$, and $\mathrm{FP}_{3 b}$, with the former and latter featuring a slightly too large top quark mass (in both cases $y_{t}$ emerges from an irrelevant UV fixed point), and the middle one instead providing a good fit to the top mass $\left(y_{t}^{*}=0\right.$ is relevant in the UV) and NP predictions closely aligned with those of $\mathrm{FP}_{2 b}$. The trans-Planckian flow for case $\mathrm{FP}_{1 c}$ is depicted in Fig. 3a, whereas the one for $\mathrm{FP}_{2 c}$ is shown in Fig. 3b. Note the presence of two subsequent IR fixed points: at the center of the plots one can see the cross-over towards the $\mathrm{FP}_{b}$-like fixed points, whereas close to the Planck scale towards FPIR.

\subsection{Low-scale predictions}

We show in Fig. 4 the LQ mass and coupling position in the plane $\left(m_{S_{3}}, \hat{Y}_{22}^{L}\right)$, predicted to be in $2 \sigma$ agreement [91] with the $b \rightarrow s$ anomalies in $\mathrm{FP}_{1 b}$ (dashed blue) and $\mathrm{FP}_{2 b}$ (solid red). The case $\mathrm{FP}_{3 b}$ roughly overlaps with $\mathrm{FP}_{1 b}$ and is not shown in the plot. The same can be said for $\mathrm{FP}_{1 c}$, $\mathrm{FP}_{3 c}$, which roughly overlap with $\mathrm{FP}_{1 b}$, and for $\mathrm{FP}_{2 c}$, which overlaps with $\mathrm{FP}_{2 b}$. In dark gray we show the current lower bound from LQ pair production at ATLAS [103], and in light gray the bound from single quark production as recast in Ref. [101]. The dashed (dotted) line marks the estimated reach of the hadron-hadron collider FCC-hh at $\sqrt{s}=100 \mathrm{TeV}$ and $1 \mathrm{ab}^{-1}\left(10 \mathrm{ab}^{-1}\right)$ integrated luminosity [104]. While the predicted LQ mass appears to be hopelessly too large to be in reach of the LHC, it fall squarely within the expected early reach of a hadron $100-\mathrm{TeV}$ machine.

The coupling $\hat{Y}_{12}^{L}-$ not included in the fixed-point analysis - is strongly constrained by the measurement of the

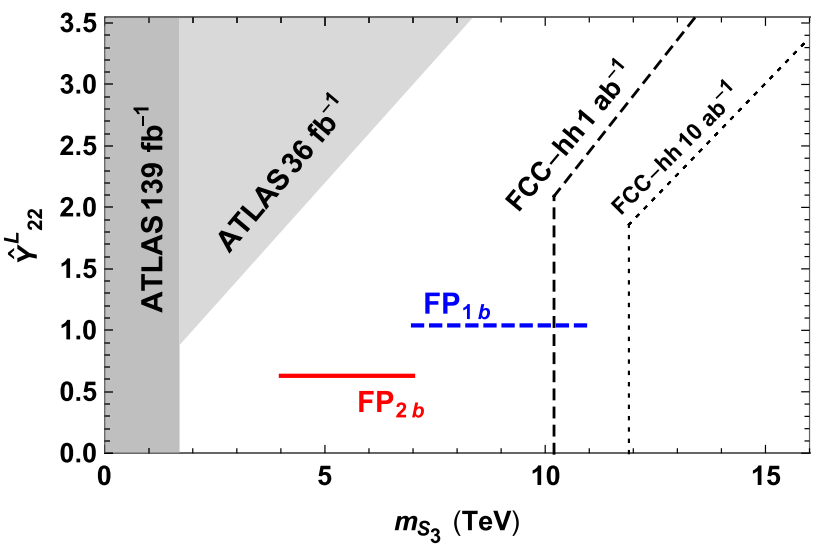

Fig. 4 The low-energy Yukawa coupling and mass ranges predicted by the trans-Planckian fixed-point analysis for the LQ $S_{3}$ to be in $2 \sigma$ agreement with the $b \rightarrow s$ anomalies. In red solid the range for $\mathrm{FP}_{2 b}$, in dashed blue the range for $\mathrm{FP}_{1 b}$. Dark gray region shows the current lower bound from color LQ pair-production at ATLAS [103], light gray region gives the bound from single quark production as recast in Ref. [101]. The dashed (dotted) black line marks the estimated reach of the hadron-hadron collider FCC-hh at $\sqrt{s}=100 \mathrm{TeV}$ and $1 \mathrm{ab}^{-1}\left(10 \mathrm{ab}^{-1}\right)$ integrated luminosity [104]

$\mathrm{BR}\left(K_{L}^{0} \rightarrow \mu^{+} \mu^{-}\right)=(6.84 \pm 0.11) \times 10^{-9}$ [105], which is practically saturated by the absorptive long-distance contribution through $K_{L}^{0} \rightarrow \gamma \gamma$. One gets the $90 \%$ C.L. bound [106]

$\left|\operatorname{Re}\left(\hat{Y}_{12}^{L *} \hat{Y}_{22}^{L}\right)\right| \lesssim 1.2 \times 10^{-5}\left(\frac{m_{S_{3}}}{\mathrm{TeV}}\right)^{2}$.

In $\mathrm{FP}_{2 b}$, the scenario in best agreement with the experimental bounds, $\hat{Y}_{32}^{L}$ and $\hat{Y}_{22}^{L}$ flow along irrelevant directions. The latter in particular becomes substantial at the low scale, being tied to the flow of the CKM matrix element $V_{33}$. If we assume that $\hat{Y}_{12}^{L}\left(M_{\mathrm{Pl}}\right)=0$ (independently of whether this happens along a relevant or irrelevant direction of flow), we get that 
$\frac{d \hat{Y}_{12}^{L}}{d t} \simeq-\frac{y_{t}^{2} V_{31}}{16 \pi^{2}}\left(V_{32} \hat{Y}_{22}^{L}+V_{33} \hat{Y}_{32}^{L}\right)$

predicts $\hat{Y}_{12}^{L}=1.6 \times 10^{-6}$ at $Q=m_{S_{3}}$, which makes this scenario consistent with Eq. (21).

Another potential low-energy bound comes from the experimental determination of the $D_{0} \rightarrow \mu^{+} \mu^{-}$branching ratio. The current measurement, $\operatorname{BR}\left(D_{0} \rightarrow \mu^{+} \mu^{-}\right)<$ $7.6 \times 10^{-9}$ at the $95 \%$ C.L. at LHCb [107] is already a few years old and might possibly be renewed with fresh data soon. Roughly following, e.g., the analysis of Ref. [108], we can express the branching ratio in terms of the $S_{3}$ LQ parameters, assuming the $t$-channel exchange of the scalar $\phi_{1 / 3}$. One gets

$$
\begin{aligned}
& \operatorname{BR}\left(D_{0} \rightarrow \mu^{+} \mu^{-}\right) \\
& \quad=\tau_{D} \frac{f_{D}^{2} M_{D}^{5}}{256 \pi M_{c}^{2}}\left(\frac{2 M_{\mu} M_{c}}{M_{D}^{2}} \frac{\lambda}{1-\lambda^{2} / 2} \frac{\left|\widetilde{Y}_{22}^{L}\right|^{2}}{2 m_{S_{3}}^{2}}\right)^{2},
\end{aligned}
$$

where $\tau_{D}=4.1 \times 10^{-13} \mathrm{~s}$ is the $D_{0}$ lifetime, $f_{D}=212 \mathrm{MeV}$ is the $D_{0}$ decay constant, $\lambda=0.226$ is a Wolfenstein parameter, and $M_{D}, M_{c}, M_{\mu}$, are the $D$ meson, charm quark, and muon mass, respectively. Equation (23) yields the $2 \sigma$ bound

$\left|V_{22} \hat{Y}_{22}^{L}\right| \lesssim 0.63 \frac{m_{S_{3}}}{\mathrm{TeV}}$,

which is currently not testing our scenarios at $Q=m_{S_{3}} \approx$ $5 \mathrm{TeV}$.

\section{Flavor anomalies in $b \rightarrow c$ transitions}

We move on to the second group of flavor anomalies receiving widespread attention in recent years: the deviations from the SM in the $R_{D^{(*)}}=\mathrm{BR}\left(\bar{B} \rightarrow D^{(*)} \tau v\right) / \operatorname{BR}\left(\bar{B} \rightarrow D^{(*)} l v\right)$ ratios, which have been observed at BELLE and LHCb [5764], confirming previous hints from BaBar [55,56]. These anomalies in $b \rightarrow c$ transitions also imply a potential violation of lepton-flavor universality and admit an explanation with LQs [65,96,98,101,109-119].

While the NP potentially contributing to the $b \rightarrow s$ anomalies has to compete with SM loop effects, in the case of the charged-current $B$ anomalies that we discuss in this section the eventual presence of NP has to compete with the $\mathrm{SM}$ at the tree level. For equivalent Yukawa couplings, new states are thus naturally expected to be much lighter than in Sect. 3, potentially in reach of the next round of LHC data. In this regard, we do not attempt in this work to analyze NP models providing a simultaneous explanation to both the $b \rightarrow s$ and $b \rightarrow c$ anomalies, but rather keep Sects. 3 and 4 separated and independent of one another. Moreover, we do not expect the trans-Planckian fixed-point analysis to provide in the case of $b \rightarrow c$ anomalies phenomenological information that is fundamentally enriching with respect to the well known findings of the numerous global fits existing in the literature $[114,118,120-125]$. We rather use this section to analyze the extent of the consistency of this NP with a gravity UV completion.

Several different operators in the weak effective theory are able to provide, alone and in combination, a $2 \sigma$ explanation to the $b \rightarrow c$ anomalies, including the vector operator $\mathscr{O}_{V_{1}}=\left(\bar{c} \gamma^{\mu} P_{L} b\right)\left(\bar{\tau} \gamma_{\mu} P_{L} \nu\right)$, which is favored in singleoperator scenarios and in combination with others. $\mathscr{O}_{V_{1}}$ can be generated at the tree level by integrating out the scalar LQ $S_{1}$ or the vector LQ $U_{1}$. As we have mentioned in Sect. 1, we focus on the scalar LQ to avoid having to introduce a further UV completion besides gravity.

The LQ $S_{1}$ is an $\mathrm{SU}(2)$ singlet. With respect to the $\mathrm{SU}(3)_{c} \times \mathrm{SU}(2)_{L} \times \mathrm{U}(1)_{Y}$ gauge group its quantum numbers are $(\overline{\mathbf{3}}, \mathbf{1}, 1 / 3)$. One writes down the interaction Lagrangian in the SM quark mass basis in terms of left-chiral (unbarred) and right-chiral (barred) two-component spinors,

$\mathscr{L} \supset\left(\widetilde{Y}_{i j}^{L} u_{L, i} e_{L, j}-\hat{Y}_{i j}^{L} d_{L, i} v_{L, j}+Y_{i j}^{R} \bar{u}_{R, i} \bar{e}_{R, j}\right) S_{1}+$ H.c.,

where repeated indices $i, j$ are summed over the SM generations, and the Yukawa coupling matrices are again related to each other by the CKM matrix $V, \widetilde{Y}_{i j}^{L}=V_{i r}^{*} \hat{Y}_{r j}^{L}$. Additional details of the $S_{1}$ model are given in Appendix B.

When integrated out at its mass scale, $S_{1}$ generates the $C_{V_{1}}$ Wilson coefficient,

$C_{V_{1}}=\frac{\hat{Y}_{33}^{L} \widetilde{Y}_{23}^{L}}{4 \sqrt{2} G_{F} V_{23} m_{S_{1}}^{2}}$,

which features up- and down-like LQ Yukawa couplings (we limit ourselves to the case of real couplings). Global fits including the ratio $R(J / \psi)$ and the longitudinal polarization fraction of the $\tau$ lepton and $D^{*}$ meson in the data set $[114,118]$ point to the $2 \sigma$ interval

$0.13\left(\frac{m_{S_{1}}^{2}}{\mathrm{TeV}^{2}}\right) \lesssim \hat{Y}_{33}^{L} \widetilde{Y}_{23}^{L} \lesssim 0.36\left(\frac{m_{S_{1}}^{2}}{\mathrm{TeV}^{2}}\right)$.

We anticipate at this point that the fixed-point analysis predicts a product of left-chiral couplings $\hat{Y}_{33}^{L} \widetilde{Y}_{23}^{L}$ too small to fall within the interval (27), without finding at the same time $m_{S_{1}}$ in the region already excluded by the LHC. It bears resemblance in this with the typical Yukawa values provided in Eqs. (18)-(20). We must therefore seek for an alternative solution, also favored by the global fits, characterized by a much smaller Wilson coefficient $C_{V_{1}}$, as long as it is accompanied by a substantial Wilson coefficient for the operator $\mathscr{O}_{S_{2}}=\left(\bar{c} P_{R} b\right)\left(\bar{\tau} P_{L} v\right), C_{S_{2}} \gtrsim 0.05$ (see, e.g., Ref. [118]). In the $S_{1}$ LQ scenario we can generate

$C_{S_{2}}=-\frac{\hat{Y}_{33}^{L} Y_{23}^{R}}{4 \sqrt{2} G_{F} V_{23} m_{S_{1}}^{2}}$,

which now involves the right-chiral couplings. 
An additional complication arises from the fact that with substantial couplings of left and right chirality that connect the charm quark to the tau lepton we generate corrections to the mass of these two particles. The Yukawa couplings of the charm quark and of the tau lepton must therefore be included in the fixed point analysis, and one ought to make sure that the corresponding masses are matched at the low energy. Note that the mass of the charm quark and the tau lepton are not very dissimilar from one another and therefore the corresponding Yukawa couplings do not feature a large hierarchy. This is fortunate, as it allows us to find, after running to the low scale, solutions that can match to the correct masses to a good approximation.

\subsection{Fixed-point analysis}

We employ the down-origin LQ Yukawa basis for the fixedpoint analysis, as justified by the low-scale phenomenology. While the fit to the $b \rightarrow c$ anomalies, Eq. (27), does not provide explicit guidance in this regard, some other strong flavor constraints do. For example, the measurement of the branching ratio $\mathrm{BR}\left(K^{+} \rightarrow \pi^{+} \nu \bar{\nu}\right)<1.85 \times 10^{-10}$ at the $90 \%$ C.L. at NA62 $[106,126]$, implies $\hat{Y}_{13}^{L} \hat{Y}_{23}^{L} \in[-3.7,8.3] \times 10^{-4}$ $\left(m_{S_{1}} / \mathrm{TeV}\right)^{2}$. In an up-type origin scenario for the couplings, this bound forces $\widetilde{Y}_{23}^{L}$ into a narrow interval, $\left(\widetilde{Y}_{23}^{L}\right)^{2} \approx$ $[-4,2] \times 10^{-3}\left(m_{S_{1}} / \mathrm{TeV}\right)^{2}$, which ends up excluding potentially viable fixed-point solutions. Conversely, the points presented in the following paragraphs, obtained in the downorigin basis for the LQ Yukawa couplings, are not in tension with flavor constraints.

The minimal set of couplings whose fixed point structure we are going to analyze consists of 12 independent parameters,

$g_{Y}, g_{2}, g_{3}, y_{t}, y_{b}, y_{c}, y_{\tau}, \hat{Y}_{23}^{L}, \hat{Y}_{33}^{L}, Y_{23}^{R}, Y_{33}^{R}, V_{33}$.

The RGEs for the $S_{1}$ plus SM system in the trans-Planckian regime are presented in Appendix B. Note that the element $Y_{33}^{R}$ of the right-handed LQ Yukawa matrix must be included in the analysis, as it is generated through the running even if its initial value is set to zero.

In analogy with the fixed-point structure discussed in Sect. 3, the non-abelian gauge couplings remain asymptotically free, while the abelian one develops a UV interactive fixed point,

$g_{3}^{*}=0, \quad g_{2}^{*}=0, \quad g_{Y}^{*}=\frac{12 \pi}{5} \sqrt{\frac{2 f_{g}}{5}}$.

By fitting to the low-scale value of the hypercharge coupling one obtains $f_{g}=0.01$ and $g_{Y}^{*}=0.48$.

The situation in the Yukawa sector of the SM is, however, more involved. As the analysis includes the RGEs of the second and third generation of up-type quarks, we strive to preserve the hierarchy $y_{t}>y_{c}$ along the full RG flow to avoid the poles in the beta function of the CKM matrix element $V_{33}$, cf. Eq. (B.39) and Ref. [37]. This implies that only three combinations of fixed-point values for top and charm Yukawa couplings are possible: $y_{t}^{*}=0, y_{c}^{*}=0 ; y_{t}^{*} \neq 0, y_{c}^{*}=0$; $y_{t}^{*}>y_{c}^{*} \neq 0$. The first of these cases does not yield solutions with LQ Yukawa couplings of the correct sign to fit the $b \rightarrow c$ anomalies. We thus focus on the cases with $y_{t}^{*} \neq 0$.

Another difference with the analysis of Sect. 3 is that we here need to fit four SM Yukawa couplings simultaneously. Like in Sect. 3, matching $y_{t}$ and $y_{b}$ to their low-scale value determines the gravitational parameter $f_{y}$, so that we should demand

$y_{c}^{*}=0, \quad y_{\tau}^{*}=0$,

and look for a solution in which both associated directions are relevant. Additionally, $y_{t}$ and $y_{c}$ generate additive chiral symmetry-breaking contributions to the tau lepton Yukawa running,

$\frac{d y_{\tau}}{d t} \approx \frac{-6}{16 \pi^{2}}\left(y_{c} \hat{Y}_{23}^{L} Y_{23}^{R}+y_{t} \hat{Y}_{33}^{L} Y_{33}^{R}\right)$

cf. Eq. (B.34). Therefore, in order to generate a UV fixed point for $y_{\tau}$ one of the elements $Y_{33}^{R}, \hat{Y}_{33}^{L}$ has to vanish at the fixed point. Since $Y_{33}^{R}$ does not enter directly the Wilson coefficients $C_{V_{1}}$ and $C_{S_{2}}$, we set $Y_{33}^{R *}=0$.

We are left with two possible combinations of fixed-point values in the SM Yukawa sector,

$$
\begin{array}{ll}
\mathrm{FP}_{1}: y_{t}^{*} \neq 0, & y_{b}^{*}=0, \\
\mathrm{FP}_{2}: y_{t}^{*} \neq 0, & y_{b}^{*} \neq 0,
\end{array}
$$

accompanied by four different choices for the LQ Yukawa matrix elements,

$$
\begin{array}{lll}
\mathrm{FP}_{a}: \hat{Y}_{23}^{L *} \neq 0, & \hat{Y}_{33}^{L *}=0, & Y_{23}^{R *} \neq 0, \\
\mathrm{FP}_{b}: \hat{Y}_{23}^{L *} \neq 0, & \hat{Y}_{33}^{L *}=0, & Y_{23}^{R *}=0, \\
\mathrm{FP}_{c}: \hat{Y}_{23}^{L *}=0, & \hat{Y}_{33}^{L *} \neq 0, & Y_{23}^{R *} \neq 0, \\
\mathrm{FP}_{d}: \hat{Y}_{23}^{L *}=0, & \hat{Y}_{33}^{L *} \neq 0, & Y_{23}^{R *}=0 .
\end{array}
$$

Note that we do not obtain any solutions corresponding to $\hat{Y}_{23}^{L *} \neq 0$ and $\hat{Y}_{33}^{L *} \neq 0$, as they are not compatible with a relevant direction for $V_{33}$.

The fixed points that are of phenomenological interest as possible solution to the $b \rightarrow c$ anomalies are summarized in Table 2. We do not report in there fixed points of the type of $\mathrm{FP}_{2}$ in Eq. (33), as they predict too large a bottom mass at the low scale.

Fixed points of type $\mathrm{FP}_{1 c}$ and $\mathrm{FP}_{1 d}$ yield a low-scale set of solutions not dissimilar to $\mathrm{FP}_{1 b}$ of Sect. 3. The dominant contribution to $\mathrm{RG}$ running takes in both cases the same form,

$\frac{d \hat{Y}_{33}^{L}}{d t} \simeq \frac{1}{16 \pi^{2}} \hat{Y}_{23}^{L} y_{t}^{2} V_{33} \sqrt{1-V_{33}^{2}}$, 
Table 2 Possible fixed points and the corresponding $f_{y}$ of the $\mathrm{SM}+S_{1}$ system with the left- and right-handed couplings. The low-energy prediction for the NP sector is shown in the last column on the right

\begin{tabular}{llllllll}
\hline & $f_{y}$ & $y_{t}^{*}$ & $y_{b}^{*}$ & $\hat{Y}_{23}^{L *}$ & $\hat{Y}_{33}^{L *}$ & $Y_{23}^{R *}$ & Fit quality at $m_{S_{1}}$ \\
\hline $1 a$ & 0.0037 & $4 \pi \frac{\sqrt{362 f_{g}+1500 f_{y}}}{5 \sqrt{295}}$ & 0 & $4 \pi \frac{\sqrt{-249 f_{g}+1250 f_{y}}}{5 \sqrt{295}}$ & 0 & $4 \pi \frac{\sqrt{68 f_{g}+65 f_{y}}}{\sqrt{295}}$ & $2 \sigma$ \\
$1 b$ & 0.0017 & $4 \pi \frac{\sqrt{14\left(27 f_{g}+125 f_{y}\right)}}{5 \sqrt{355}}$ & 0 & $4 \pi \frac{\sqrt{219 f_{g}+2000 f_{y}}}{5 \sqrt{355}}$ & 0 & 0 & $\hat{Y}_{33}^{L} \hat{Y}_{23}^{L}<0$ \\
$1 c$ & 0.0032 & $4 \pi \frac{\sqrt{51 f_{g}+250 f_{y}}}{15 \sqrt{5}}$ & 0 & 0 & $2 \pi \frac{\sqrt{-66 f_{g}+500 f_{y}}}{25}$ & $-4 \pi \frac{\sqrt{141 f_{g}+125 f_{y}}}{25}$ & $\hat{Y}_{33}^{L} \hat{Y}_{23}^{L}$ too small \\
$1 d$ & 0.0044 & $4 \pi \frac{\sqrt{51 f_{g}+250 f_{y}}}{15 \sqrt{5}}$ & 0 & 0 & $2 \pi \frac{\sqrt{3 f_{g}+25 f_{y}}}{5}$ & 0 & $\hat{Y}_{33}^{L} \hat{Y}_{23}^{L}$ too small \\
\hline
\end{tabular}

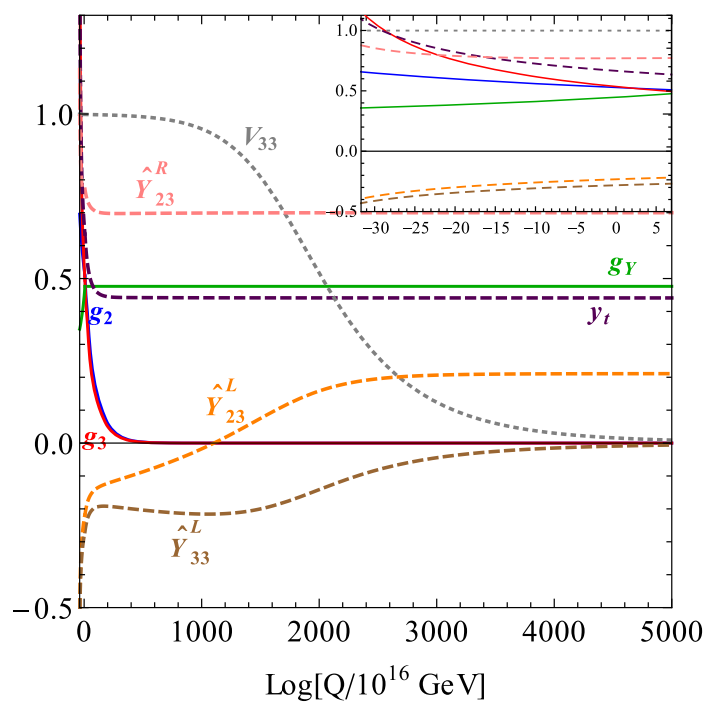

Fig. 5 RG flow of the gauge and Yukawa couplings from the transPlanckian energies down to the EWSB scale in the case of scenario $\mathrm{FP}_{1 a}$. The sub-Planckian flow is depicted in the inset panels

which leads to a reduction of the matrix element $\hat{Y}_{33}^{L}$ during its flow towards the IR. The low-energy value of $\hat{Y}_{33}^{L}$ is very small and results inconsistent with either Eq. (27) or $C_{S_{2}} \gtrsim 0.05$ at the TeV scale (the low-scale value we obtain for $Y_{23}^{R}$ does not affect this conclusion).

We now turn to discussing scenarios $\mathrm{FP}_{1 a}$ and $\mathrm{FP}_{1 b}$. The asymptotically free coupling $\hat{Y}_{33}^{L}$ is now associated with a relevant direction and its deviation from the UV fixed point is a free parameter of the theory. Since the main contribution to its running is given by Eq. (35), it is driven to negative values as soon as the CKM matrix element $V_{33}$ starts to depart from its fixed point. The RG flow of the coupling system from the vicinity of the UV fixed point towards the IR is shown for $\mathrm{FP}_{1 a}$ in Fig. 5.

Fixed points $\mathrm{FP}_{1 a}$ and $\mathrm{FP}_{1 b}$ bear resemblance to $\mathrm{FP}_{1 a}$ of Sect. 3, see also Fig. 1a. They do not, however, yield an identical low-scale phenomenology. The evolution of $\mathrm{FP}_{1 a}$ is triggered mostly by the presence of the right-handed irrel- evant coupling $Y_{23}^{R}$. Since its contribution to the running of $\hat{Y}_{23}^{L}$ is large and positive,

$\frac{d \hat{Y}_{23}^{L}}{d t} \simeq \frac{1}{16 \pi^{2}}\left(\hat{Y}_{23}^{L}\left(\hat{Y}_{23}^{R}\right)^{2}-2 \hat{Y}_{33}^{L} y_{t}^{2} V_{33} \sqrt{1-V_{33}^{2}}\right)$,

see also Eq. (B.35), $\hat{Y}_{23}^{L}$ decreases towards the IR. As a consequence, we see that in $\mathrm{FP}_{1 a}$ both $\hat{Y}_{23}^{L}$ and $\hat{Y}_{33}^{L}$ can become negative and relatively sizable at the Planck scale. This behavior guarantees the partial consistency of the low-energy predictions of the model with the $b \rightarrow c$ flavor anomalies. Conversely, in scenario $\mathrm{FP}_{1 b} \hat{Y}_{23}^{L}$ never becomes negative due to a small fixed-point value of the top Yukawa coupling. As a consequence, $\mathrm{FP}_{1 b}$ turns out to be not consistent with the low-energy phenomenology of the flavor anomalies.

The Yukawa couplings $y_{c}, y_{b}, y_{\tau}$, which vanish at the fixed point, correspond to relevant directions in the coupling space, as long as $f_{y}$ exceeds the minimal value for which $y_{b}$ becomes relevant, as discussed in Sect. 3. We checked that by fixing $f_{y}$ at this minimal value we predict the top mass at $Q=m_{S_{1}}=1.5 \mathrm{TeV}$ in very good agreement with the experimental measurement, $y_{t}\left(m_{S_{1}}\right)=0.98$. However, the elements $\hat{Y}_{33}^{L}\left(m_{S_{1}}\right), \hat{Y}_{23}^{L}\left(m_{S_{1}}\right)$, and $\hat{Y}_{23}^{R}\left(m_{S_{1}}\right)$ are too small to fit the $b \rightarrow c$ anomalies. Therefore, we need to increase $f_{y}$ at the price of enhancing the top mass. Choosing $f_{y}=0.0036$, we obtain the following predictions for $\mathrm{FP}_{1 a}$ :

$$
\begin{array}{cl}
y_{t}\left(m_{S_{1}}\right)=1.01, & y_{b}\left(m_{S_{1}}\right)=0.013, \\
y_{c}\left(m_{S_{1}}\right)=-0.009, & y_{\tau}\left(m_{S_{1}}\right)=0.01, \\
\hat{Y}_{23}^{L}\left(m_{S_{1}}\right)=-0.37, & \hat{Y}_{33}^{L}\left(m_{S_{1}}\right)=-0.41 . \\
Y_{23}^{R}\left(m_{S_{1}}\right)=0.85 . &
\end{array}
$$

In all the scenarios discussed in this section $y_{t}$ corresponds to an irrelevant direction and the low-scale prediction for the top mass results by $\sim 10 \%$ too large with respect to its experimentally measured value. We also observe that a fit to the tau lepton mass drives the charm mass to values by $40 \%$ too large, even if the corresponding Yukawa couplings flow along relevant directions. This is an expected effect, 


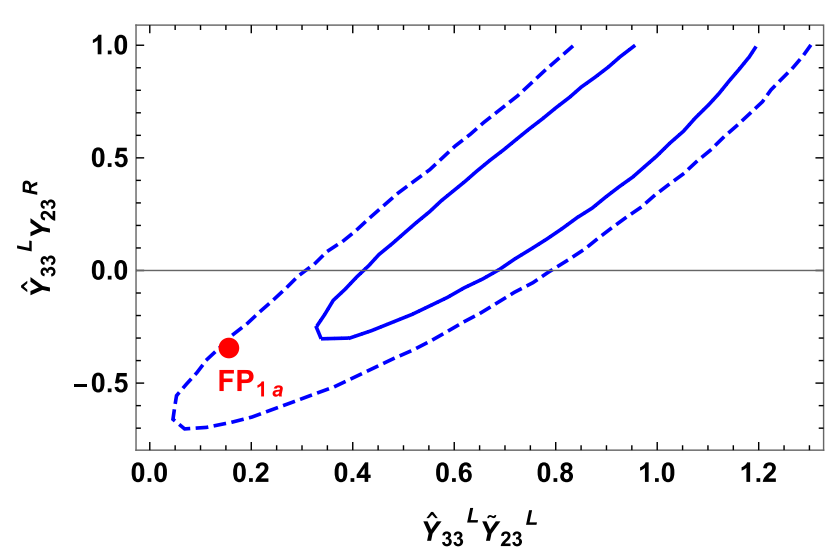

Fig. 6 Solid (dashed) blue line gives the $1 \sigma(2 \sigma)$ confidence region of the $b \rightarrow c$ global fit of Ref. [118] in the plane $\left(\hat{Y}_{33}^{L} \widetilde{Y}_{23}^{L}, \hat{Y}_{33}^{L} Y_{23}^{R}\right)$ of the $S_{1}$ Yukawa couplings at $m_{S_{1}}=1.5 \mathrm{TeV}$. The red dot marks the position of $\mathrm{FP}_{1 a}$

due to the explicit breaking of chiral symmetry introduced by the presence of the $Y_{i j}^{R}$ couplings, which ties the running of $y_{c}$ to $y_{\tau}$. All these things considered, however, the fixed point of type $\mathrm{FP}_{1 a}$ features a satisfactory agreement between the quantum gravity UV completion and the full low-scale phenomenology.

\subsection{Low-scale predictions}

We show in Fig. 6 the position of the fixed point $\mathrm{FP}_{1 a}$ in the plane $\left(\hat{Y}_{33}^{L} \widetilde{Y}_{23}^{L}, \hat{Y}_{33}^{L} Y_{23}^{R}\right)$, compared to the $1 \sigma$ (solid) and $2 \sigma$ (dashed) regions of the global fit to $R_{D} / R_{D^{*}}, R(J / \psi)$, and the longitudinal polarization of the $\tau$ and $D^{*}$ presented in Ref. [118]. Like in Ref. [118], the LQ mass is set at a reference value of $m_{S_{1}}=1.5 \mathrm{TeV}$.

Given the presence of relatively large left-handed LQ Yukawa couplings in Eq. (37), the model is subject to a tight constraint from the measurement of the branching ratio $\operatorname{BR}\left(B \rightarrow K^{(*)} \bar{v} v\right)$ [109]. The LQ contribution can be written as [127]

$\mathscr{R}_{K}=\mathscr{R}_{K^{*}}=\frac{2}{3}+\frac{1}{3} \frac{\left|C_{L}^{\mathrm{SM}}-2 \tilde{c}_{q l}\right|^{2}}{\left|C_{L}^{\mathrm{SM}}\right|^{2}}$,

where $C_{L}^{\mathrm{SM}}=-6.38[128]$ and

$\tilde{c}_{q l} \approx \frac{(5 \mathrm{TeV})^{2}}{V_{33} V_{32}^{*}}\left(\frac{1}{4} \frac{\hat{Y}_{23}^{L} \hat{Y}_{33}^{L}}{m_{S_{1}}^{2}}\right)$

By comparing the experimental determination, $\mathrm{BR}(B \rightarrow$ $\left.K^{* 0} \bar{\nu} v\right)_{\exp }<1.8 \times 10^{-5}$ at the $90 \%$ C.L. [129], with $\mathscr{R}_{K^{*}} \cdot \mathrm{BR}\left(B \rightarrow K^{* 0} \bar{v} v\right)_{\mathrm{SM}}$, where $\mathrm{BR}\left(B \rightarrow K^{* 0} \bar{v} v\right)_{\mathrm{SM}}=$
$0.92 \times 10^{-5}$, one gets

$$
-0.020 \leq \frac{\hat{Y}_{23}^{L} \hat{Y}_{33}^{L}}{m_{S_{1}}^{2} / \mathrm{TeV}^{2}} \leq 0.061,
$$

which places $\mathrm{FP}_{1 a}$ at the very edge of exclusion. Note that the model parameters imply that $\mathscr{R}_{K^{*}}=\mathscr{R}_{K}=2.4$ and that all decays of the type $\operatorname{BR}\left(B \rightarrow K^{(*)} \bar{v} v\right)$ provide an equally good experimental venue to probe this kind of models.

A potentially complementary signature for testing this scenario can be obtained by improving the precision of lepton universality measurements in the kaon sector. We define the universality as

$$
R_{\tau / \mu}^{K, \exp }=\frac{\mathrm{BR}\left(\tau^{-} \rightarrow K^{-} \nu\right)}{\mathrm{BR}\left(K^{-} \rightarrow \mu^{-} \nu\right)}=0.01095 \cdot(1 \pm 0.015),
$$

as reported by the Particle Data Group (PDG) [130]. In the $\mathrm{FP}_{1 a}$ case we calculate

$$
R_{\tau / \mu}^{K}=\frac{\tau_{\tau}}{\tau_{K}} \frac{r_{K}^{3}}{2 r_{\mu}^{2}}\left(\frac{1-r_{K}^{2}}{r_{K}^{2}-r_{\mu}^{2}}\right)^{2} \frac{\left(V_{12}+g_{L}\right)^{2}}{V_{12}^{2}},
$$

where $\tau_{\tau}=2.9 \times 10^{-13} \mathrm{~s}$ and $\tau_{K}=1.25 \times 10^{-8} \mathrm{~s}$ are the tau and kaon lifetimes, $V_{12} \approx \lambda \approx 0.226, r_{X}=M_{X} / M_{\tau}$, and

$g_{L} \equiv \frac{v_{h}^{2}}{m_{S_{1}}^{2}} \frac{\lambda\left(\hat{Y}_{23}^{L}\right)^{2}}{4}$

in terms of the Higgs vev, $v_{h}$. At $1 \sigma$ we get

$\left|\hat{Y}_{23}^{L}\right| \leq 0.57 \frac{m_{S_{1}}}{\mathrm{TeV}}$.

To test the value derived in Eq. (37), $\hat{Y}_{23}^{L}=-0.37$ at $m_{S_{1}}=$ $1.5 \mathrm{TeV}$, the measurement error in Eq. (41) should be reduced to $\delta R_{\tau / \mu}^{K} / R_{\tau / \mu}^{K} \approx 2 \times 10^{-3}$.

Besides $R_{\tau / \mu}^{K}$, similar sensitivity to the $S_{1}$ model is expected in the observables $R_{\tau / \mu}^{D_{s}}$ and $B \rightarrow \tau \nu$, which provide additional testing ground for this scenario.

Finally, one can use well known analytic approximations (see, e.g., Ref. [114]) to calculate $R_{D}$ and $R_{D^{*}}$ given the parameters of $\mathrm{FP}_{1 a}$ in Eq. (37). With $m_{S_{1}}=1.5 \mathrm{TeV}$ we obtain

$$
R_{D}\left(\mathrm{FP}_{1 a}\right)=0.341, \quad R_{D^{*}}\left(\mathrm{FP}_{1 a}\right)=0.270 .
$$

On the other hand, strictly speaking $m_{S_{1}}=1.5 \mathrm{TeV}$ is already excluded at the $95 \%$ C.L. by the most recent ATLAS data on LQ pair production in the gluon-gluon channel [103]. The most recent bound, at $m_{S_{1}}>1.7 \mathrm{TeV}$, pushes $\mathrm{FP}_{1 a}$ slightly outside of the $2 \sigma$ favored region for the $R_{D^{(*)}}$ anomalies, as the contours in Fig. 6 move a little to the right and down, whereas the benchmark point remains roughly in the same place. Incidentally, at $m_{S_{1}}=1.7 \mathrm{TeV}$ the constraint from 
$\mathrm{BR}\left(B \rightarrow K^{* 0} \bar{v} v\right)$ falls short of excluding the Yukawa values given in Eq. (37). One recalculates

$$
\begin{aligned}
& R_{D}\left(\mathrm{FP}_{1 a}, 1.7 \mathrm{TeV}\right)=0.332 \\
& R_{D^{*}}\left(\mathrm{FP}_{1 a}, 1.7 \mathrm{TeV}\right)=0.267 .
\end{aligned}
$$

At $m_{S_{1}}=1.7 \mathrm{TeV}$, the LHC is expected to reach in the $p p \rightarrow \mu^{+} \mu^{-}$jet channel (quark-quark + quark-gluon production) a $95 \%$ C.L. sensitivity to $\left|Y_{22}^{R}\right| \approx 0.80$ with $300 \mathrm{fb}^{-1}$ of integrated luminosity [108]. In the corresponding channel with final-state tau leptons - appropriate to test the value given in Eq. (37), $Y_{23}^{R}=0.85$ - the LHC sensitivity drops, and is expected to exclude a Yukawa coupling larger by approximately $60 \%$ than in the muon case [101]. Still, a combination of the reach of gluon-gluon, quark-quark, quark-gluon production channels is likely to corner the scenario discussed here in the very near future.

\section{Summary and conclusions}

In this paper we used the framework of asymptotically safe quantum gravity to derive predictions for the mass of scalar LQs as solutions to the experimental anomalies recorded in recent years in $b \rightarrow s$ and $b \rightarrow c$ transitions. Our predictions are obtained by embedding a SM extension with a single LQ in a trans-Planckian completion in which gravitational interactions induce corrections to the beta functions of the Lagrangian (gauge and Yukawa) couplings. The latter thus develop interactive fixed points in the extreme UV, which parametrize specific sets of boundary conditions at the Planck scale. The flavor phenomenology is then unambiguously determined by following the coupling flow to the EWSB scale.

The low-scale phenomenological predictions follow from two main sources: the presence of a fixed point in $g_{Y}$ (and therefore the size and sign of $f_{g} \approx 0.01$ ) and the decoupling scale $M_{\mathrm{Pl}}$. In this sense, the predictions described here can potentially stem from quantum gravity effects as long as they induce an interactive trans-Planckian fixed point in $g_{Y}$ that can be matched to the SM. The leading-order correction to the Yukawa couplings, $f_{y}$, has subdominant impact on the final result (additionally, $f_{y}$ cancels out altogether from the beta function of $V_{33}$ ). Note that our low-scale predictions do not depend on the higher-order expansion of the physics above the Planck scale, but only on the Planck-scale value of the SM and NP couplings.

Being flavor-blind, gravitational interactions do not generate by their own very nature flavor signatures. They can, however, be a source for the Planck-scale boundary conditions of the Yukawa textures favored by the flavor phenomenology. Once the low-scale value of the NP Yukawa couplings is determined in this way, we found that by assuming a $2 \sigma$ con- sistency with the neutral-current, $b \rightarrow s$ anomalies, the predicted LQ mass lies in the range $4-7 \mathrm{TeV}$. These values are too large to be in reach of the high-luminosity LHC, but fall squarely within the early reach of a $100-\mathrm{TeV}$ hadron collider, according to the most conservative estimates. Complementary signatures in flavor observables like $\mathrm{BR}\left(K_{L} \rightarrow \mu^{+} \mu^{-}\right)$ or $\operatorname{BR}\left(D_{0} \rightarrow \mu^{+} \mu^{-}\right)$require significant increases in the experimental sensitivity with respect to the current bounds.

The trans-Planckian fixed-point analysis encounters some additional complications when applied to LQ solutions to the charged-current, $b \rightarrow c$ anomalies, mostly due to the presence of explicit chiral-symmetry violating terms in the Yukawa coupling RGEs, which induce some tension in the low-energy fit to the fermion masses. The situation is, however, very optimistic from the observational point of view, as the LQ mass and Yukawa couplings are predicted to be at the very edge of the current LHC bounds, well within the reach of $300 \mathrm{fb}^{-1}$.

The present study can be extended in several directions. First of all, the fixed-point analysis can be broadened to incorporate the impact of all three families of quarks and three real mixing angles of the CKM matrix. While we do not expect this extension to affect significantly our predictions on the low-energy NP phenomenology, it would be interesting to verify whether such a setup can still be consistent with the SM.

In a similar spirit, one could include in the analysis the non-trivial flavor structure of the leptonic sector, which we neglected here for the sake of simplicity. The non-trivial form of the neutrino mixing matrix could potentially lead to lepton flavor-changing signatures, which will be tested in the next few years by experiments like MEG-II and others. Following this direction of investigation, one would also need to address the mechanism for generating neutrino masses.

Finally, we did not incorporate in the fixed-point analysis the parameters characterizing the scalar potential of the model. We do not expect them to impact our findings, as the scalar couplings enter the RGEs of the gauge and Yukawa couplings at the third and the second loop, respectively, and in the perturbative domain their impact becomes highly loop-suppressed. However, an extended analysis of the scalar potential can be an interesting topic per se. The issue of its stability, as well as the correct prediction of the Higgs boson mass, could be analyzed and compared to the NP predictions and signatures presented here.

LQ solutions to the flavor anomalies have been extensively studied in the literature from the point of view of their compatibility with a large number of constraints defined at about and below their mass scale. We find it encouraging that they can also show consistency with the theoretical framework of asymptotically safe quantum gravity, which is defined far above the scale that can be tested directly and was previously left unexplored in this context. 
Acknowledgements We would like to thank Daniel Litim for helpful comments on the manuscript. KK is supported in part by the National Science Centre (Poland) under the research Grant No. 2017/26/E/ST2/00470. EMS and YY are supported in part by the National Science Centre (Poland) under the research Grant No. 2017/26/D/ST2/00490.

Data Availability Statement This manuscript has no associated data or the data will not be deposited. [Authors' comment: The presented study has theoretical character and no data has been produced.]

Open Access This article is licensed under a Creative Commons Attribution 4.0 International License, which permits use, sharing, adaptation, distribution and reproduction in any medium or format, as long as you give appropriate credit to the original author(s) and the source, provide a link to the Creative Commons licence, and indicate if changes were made. The images or other third party material in this article are included in the article's Creative Commons licence, unless indicated otherwise in a credit line to the material. If material is not included in the article's Creative Commons licence and your intended use is not permitted by statutory regulation or exceeds the permitted use, you will need to obtain permission directly from the copyright holder. To view a copy of this licence, visit http://creativecomm ons.org/licenses/by/4.0/.

Funded by SCOAP ${ }^{3}$.

\section{Appendix A: Trans-Planckian renormalization of $S_{3}$}

We review in this appendix the notation we use for rotating the SM and LQ fields. The LQ $S_{3}$ is characterized by the SM quantum numbers $(\overline{\mathbf{3}}, \mathbf{3}, 1 / 3)$. One defines the $3 \times 3$ Yukawa matrix $Y_{L}$ in the "flavor" (or gauge-symmetric) basis according to the Lagrangian

$\mathscr{L} \supset\left(Y_{L}\right)_{i j} Q_{i}^{T}\left(i \sigma_{2}\right) S_{3} L_{j}+$ H.c.,

where $Q_{i}^{T}=\left(u_{L i}, d_{L i}\right)$ and $L_{j}=\left(v_{L j}, e_{L j}\right)^{T}$ are $\operatorname{SU}(2)_{L}$ doublets of two-component left-chiral Weyl spinors, $S_{3}$ is the scalar LQ matrix

$S_{3}=\left(\begin{array}{cc}\phi_{1 / 3} & \sqrt{2} \phi_{4 / 3} \\ \sqrt{2} \phi_{-2 / 3} & -\phi_{1 / 3}\end{array}\right)$,

and $\sigma_{2}$ is the second Pauli matrix. It is well known that the quantum numbers of $S_{3}$ allow for the presence of Lagrangian terms of the type $Q^{T}\left(i \sigma_{2}\right) S_{3}^{\dagger} Q$, potentially leading to fast proton decay [131]. We assume in this paper that these are forbidden by a symmetry (for example, conservation of baryon and/or lepton number).

The LQ is characterized by a scalar potential connecting it to the SM Higgs doublet, $H$ :

$$
\begin{aligned}
V\left(H, S_{3}\right)= & \frac{1}{2} m_{S_{3}}^{2} \operatorname{Tr}\left[S_{3}^{\dagger} S_{3}\right]+\frac{1}{8} \lambda_{S_{3}}\left(\operatorname{Tr}\left[S_{3}^{\dagger} S_{3}\right]\right)^{2} \\
& +\frac{1}{2} \lambda_{H S_{3}} \operatorname{Tr}\left[S_{3}^{\dagger} S_{3}\right] H^{\dagger} H .
\end{aligned}
$$

The LQ fields do not develop a vacuum expectation value (vev).
We carry out the transformation from the flavor basis to the quark mass basis via the unitary rotation matrices $U_{L}, U_{R}$, $D_{L}$, and $D_{R}$. If $Y_{U}, Y_{D}$, and $Y_{E}$ are the Yukawa matrices of the SM in the flavor basis, the corresponding diagonal matrices $Y_{u}$ and $Y_{d}$ in the mass basis are given by

$Y_{u}=U_{L}^{\dagger} Y_{U} U_{R}, \quad Y_{d}=D_{L}^{\dagger} Y_{D} D_{R}$,

and the CKM matrix is defined as $V \equiv U_{L}^{\dagger} D_{L}$. We assume for simplicity that the charged lepton Yukawa matrix is trivially diagonal in the flavor basis so that $Y_{e} \equiv Y_{E}$.

We work in the quark mass basis throughout this work. We further introduce several assumptions, which significantly simplify the analysis and yet produce interesting phenomenological signatures at the low scale.

- We treat the Yukawa couplings of the SM and NP as real in flavor space

- Above the LQ mass scale, we work in the 2-quark family approximation. As the flavor anomalies only constrain the second and third quark family this is a reasonable approximation simplifying the fixed-point analysis. As a direct consequence, the CKM matrix is orthogonal and described by one rotation angle for the purposes of the fixed-point analysis

- We neglect the physics of neutrino masses and oscillations. In this approximation the SM charged lepton Yukawa matrices are rotated by the identity matrix

- We restrict the fixed-point analysis to the gauge-Yukawa system at one loop. We have checked numerically that the predictions for the NP couplings change minimally under the addition of perturbative 2-loop contributions. The parameters of the scalar potential do not enter at one loop in the gauge-Yukawa RGEs, and do not affect the phenomenological predictions presented in this work. One should keep in mind, however, that if the theory is expected to be complete the full fixed-point analysis should include the parameters of Eq. (A.3) and their coupling to the graviton, $f_{\lambda}$. The full system should show consistency with the $125 \mathrm{GeV}$ Higgs mass and the stability of the scalar potential at all scales (see Refs. [132135] for some related work). We leave the analysis of these important but separated issues for future work.

One obtains the LQ Yukawa matrices introduced in Sect. 3 in the quark mass basis via unitary rotations

$\tilde{Y}^{L}=U_{L}^{T} Y_{L}, \quad \hat{Y}^{L}=D_{L}^{T} Y_{L}$,

which lead to $\widetilde{Y}^{L}=V^{*} \hat{Y}^{L}$. We emphasize that the matrices in Eq. (A.5) are not diagonal as we do not enforce any flavor symmetry.

Since the texture of the SM and NP Yukawa matrices is not fixed by additional flavor symmetries, their elements are 
all subject to RG-running modifications. Elements that are zero in a particular basis at one scale, do not necessarily remain zero or small in the same basis at a different scale. One can however choose to remain at each and every scale in one specific basis, for example the quark mass basis that we select in this work, in which the SM Yukawa matrices are diagonal and the rotation matrices run accordingly. The explicit scale dependence of $U_{L}(t), U_{R}(t), D_{L}(t)$, and $D_{R}(t)$ will have to be factored into the RG flow.

Additionally, one can choose to carry out the fixed-point analysis in a preferential basis for the LQ Yukawa matrices: the up-origin $\widetilde{Y}^{L}$, or the down-origin $\hat{Y}^{L}$. Whether one or the other basis is chosen will result in one or another set of UV fixed point. Some of the fixed points will be consistent with the low-scale phenomenological constraints, whereas others will be in tension or outright excluded. In the case of the $b \rightarrow s$ anomalies, the nature of the constraint, Eq. (8), leads to the natural choice of the down-origin basis for our analysis. Following standard procedure (see, e.g., Ref. [37]), we define the squared Yukawa matrices at the scale $t=\log Q$ in the flavor basis:

$M_{U}=Y_{U} Y_{U}^{\dagger}, \quad M_{D}=Y_{D} Y_{D}^{\dagger}, \quad M_{L}=Y_{L} Y_{L}^{\dagger}$

The corresponding diagonal Yukawa matrices in the mass basis at the scale $t$ are given by

$Y_{u}^{2}(t)=U_{L}^{\dagger}(t) M_{U}(t) U_{L}(t)$,

$Y_{d}^{2}(t)=D_{L}^{\dagger}(t) M_{D}(t) D_{L}(t)$,

as well as the non-diagonal squared matrix

$$
\left(\hat{Y}^{L} \hat{Y}^{L \dagger}\right)(t)=D_{L}^{T}(t) M_{L}(t) D_{L}^{*}(t) .
$$

We use SARAHv4 . 12 . 2 [136] to derive the one-loop RG flow for the (squared) Yukawa matrices in the flavor basis and then use the unitarity of the rotation matrices to connect them to the RG flow in the mass basis. One writes

$$
\begin{aligned}
& \partial_{t} Y_{u}^{2}+\left[Y_{u}^{2},\left(\partial_{t} U_{L}^{\dagger}\right) U_{L}\right]=U_{L}^{\dagger}\left(\partial_{t} M_{U}\right) U_{L} \\
& \partial_{t} Y_{d}^{2}+\left[Y_{d}^{2},\left(\partial_{t} D_{L}^{\dagger}\right) D_{L}\right]=D_{L}^{\dagger}\left(\partial_{t} M_{D}\right) D_{L} \\
& \partial_{t}\left(\hat{Y}^{L} \hat{Y}^{L \dagger}\right)+\left[\hat{Y}^{L} \hat{Y}^{L \dagger},\left(\partial_{t} D_{L}^{T}\right) D_{L}^{*}\right]=D_{L}^{T}\left(\partial_{t} M_{L}\right) D_{L}^{*} .
\end{aligned}
$$

The 1.h.s. of Eqs. (A.9) and (A.10) is now recast as a sum of one diagonal and one purely off-diagonal matrix, the first of which features the SM Yukawa coupling beta function, whereas the second parametrizes the scale dependence of the rotation matrices. Note, on the other hand, that the 1.h.s. of Eq. (A.11) is not characterized by any specific texture, as both addends feature diagonal and off-diagonal elements.

The scale dependence of Eqs. (A.9) and (A.10) can be used to derive unambiguously the flow of the absolute values

of the CKM matrix elements [137-140]. We use the unitarity of the rotation matrices and the fact that $V=U_{L}^{\dagger} D_{L}$ to write

$$
\frac{d V_{i j}}{d t}=\sum_{k=1,2,3}\left[\left(\partial_{t} U_{L}^{\dagger} U_{L}\right)_{i k} V_{k j}-V_{i k}\left(\partial_{t} D_{L}^{\dagger} D_{L}\right)_{k j}\right] \text {, }
$$

and then use the identity

$$
\frac{1}{\left|V_{i j}\right|^{2}} \frac{d\left|V_{i j}\right|^{2}}{d t}=2 \operatorname{Re}\left(\frac{1}{V_{i j}} \frac{d V_{i j}}{d t}\right)
$$

to be allowed to ignore safely the unknown imaginary part of the diagonal elements of the matrices $\partial_{t} U_{L}^{\dagger} U_{L}$ and $\partial_{t} D_{L}^{\dagger} D_{L}$.

We present here the one-loop gauge-Yukawa-CKM system of equations for $S_{3}$ in the down-origin basis and 2-family approximation. We only give the equations for the parameters affecting the low-scale phenomenology of the $b \rightarrow s$ anomalies. All other parameters can be considered to be zero and relevant at the UV fixed point.

$$
\begin{aligned}
& \frac{d g_{3}}{d t}=-\frac{13}{2} \frac{g_{3}^{3}}{16 \pi^{2}}-f_{g} g_{3} \\
& \frac{d g_{2}}{d t}=-\frac{7}{6} \frac{g_{2}^{3}}{16 \pi^{2}}-f_{g} g_{2} \\
& \frac{d g_{Y}}{d t}=\frac{43}{6} \frac{g_{Y}^{3}}{16 \pi^{2}}-f_{g} g_{Y} \\
& \frac{d y_{t}}{d t}=\frac{1}{16 \pi^{2}}\left[3 y_{b}^{2}+\frac{9}{2} y_{t}^{2}-\frac{17}{12} g_{Y}^{2}\right. \\
& -\frac{9}{4} g_{2}^{2}-8 g_{3}^{2}-\frac{3}{2} V_{33}^{2} y_{b}^{2} \\
& +\frac{3}{4}\left(V_{32}{ }^{2}\left(\hat{Y}_{22}^{L}\right)^{2}+2 V_{32} V_{33} \hat{Y}_{22}^{L} \hat{Y}_{32}^{L}\right. \\
& \left.\left.+V_{33}^{2}\left(\hat{Y}_{32}^{L}\right)^{2}\right)\right] y_{t}-f_{y} y_{t} \\
& \frac{d y_{b}}{d t}=\frac{1}{16 \pi^{2}}\left[\frac{9}{2} y_{b}^{2}+y_{t}^{2}-\frac{5}{12} g_{Y}^{2}\right. \\
& -\frac{9}{4} g_{2}^{2}-8 g_{3}^{2}-\frac{3}{2} V_{33}^{2} y_{t}^{2} \\
& \left.+\frac{3}{4}\left(\hat{Y}_{32}^{L}\right)^{2}\right] y_{b}-f_{y} y_{b} \\
& \frac{d \hat{Y}_{22}^{L}}{d t}=\frac{1}{16 \pi^{2}}\left\{\left[\frac{7}{2}\left(\hat{Y}_{22}^{L}\right)^{2}+\frac{11}{4}\left(\hat{Y}_{32}^{L}\right)^{2}\right.\right. \\
& -\frac{5}{6} g_{Y}^{2}-\frac{9}{2} g_{2}^{2}-4 g_{3}^{2} \\
& \left.\left.+\frac{1}{2} y_{t}^{2} V_{32}^{2}\right] \hat{Y}_{22}^{L}+2 y_{t}^{2} V_{32} V_{33} \hat{Y}_{32}^{L}\right\} \\
& -f_{y} \hat{Y}_{22}^{L} \\
& \frac{d \hat{Y}_{32}^{L}}{d t}=\frac{1}{16 \pi^{2}}\left\{\left[\frac{17}{4}\left(\hat{Y}_{22}^{L}\right)^{2}+\frac{7}{2}\left(\hat{Y}_{32}^{L}\right)^{2}\right.\right. \\
& +\frac{1}{2} y_{b}^{2}-\frac{5}{6} g_{Y}^{2}-\frac{9}{2} g_{2}^{2}
\end{aligned}
$$




$$
\begin{aligned}
& \left.\left.-4 g_{3}^{2}+\frac{1}{2} y_{t}^{2} V_{33}^{2}\right] \hat{Y}_{32}^{L}-y_{t}^{2} V_{32} V_{33} \hat{Y}_{22}^{L}\right\} \\
& -f_{y} \hat{Y}_{32}^{L} \\
\frac{d\left|V_{33}\right|}{d t}= & \frac{V_{23}}{16 \pi^{2}}\left[-\frac{3}{2} V_{23} V_{33} y_{b}^{2}+\frac{3}{4}\left(V_{22} V_{32}\left(\hat{Y}_{22}^{L}\right)^{2}\right.\right. \\
& +V_{22} V_{33} \hat{Y}_{22}^{L} \hat{Y}_{32}^{L}+V_{23} V_{32} \hat{Y}_{22}^{L} \hat{Y}_{32}^{L} \\
& \left.\left.+V_{23} V_{33}\left(\hat{Y}_{32}^{L}\right)^{2}\right)\right] \\
& -\frac{V_{32}}{16 \pi^{2}}\left[\frac{3}{2} V_{32} V_{33} y_{t}^{2}-\frac{3}{4} \hat{Y}_{22}^{L} \hat{Y}_{32}^{L}\right] .
\end{aligned}
$$

Moreover, the orthogonality of the CKM matrix in the 2family approximation leads to

$V_{22}=V_{33}, \quad V_{23}=-V_{32}=\sqrt{1-V_{33}^{2}}$.

\section{Appendix B: Trans-Planckian renormalization of $S_{1}$}

We present here the RG flow of the $S_{1}$ gauge-Yukawa system in the trans-Planckian regime. $S_{1}$ carries the SM quantum numbers $(\overline{\mathbf{3}}, \mathbf{1}, 1 / 3)$. In the flavor basis, the Lagrangian features NP Yukawa matrices of the left (L) and right (R) type. In terms of left-chiral (unbarred) and right-chiral (barred) Weyl fields one writes

$\mathscr{L} \supset\left(Y_{L}\right)_{i j} Q_{i}^{T}\left(i \sigma_{2}\right) L_{j} S_{1}+\left(Y_{R}\right)_{i j} \bar{u}_{R i} \bar{e}_{R j} S_{1}+$ H.c.,

where a sum over repeated family indices $i, j$ is implied, $Q_{i}^{T}=\left(u_{L i}, d_{L i}\right)$ and $L_{j}=\left(v_{L j}, e_{L j}\right)^{T}$ are $\mathrm{SU}(2)_{L}$ doublets and $\sigma_{2}$ is the second Pauli matrix. As was the case for $S_{3}$, we assume that terms that are dangerous for proton decay are forbidden.

In dealing with Eq. (B.23), we adopt the assumptions introduced and justified in Appendix A. Namely, we restrict ourselves in the fixed-point analysis to real Yukawa couplings and the 2-family approximation; we neglect the physics of neutrinos and consider only diagonal charged-lepton matrices; we do not introduce the scalar potential sector parameters in the fixed-point analysis.

The Yukawa matrices transform to the quark mass basis via the unitary rotations

$Y^{R}=U_{R}^{T} Y_{R} \quad \tilde{Y}^{L}=U_{L}^{T} Y_{L} \quad \hat{Y}^{L}=D_{L}^{T} Y_{L}$,

which yield $\tilde{Y}^{L}=V^{*} \hat{Y}^{L}$.

As is explained in Sect. 4, we perform the fixed-point analysis in the down-origin basis of the left-handed LQ Yukawa couplings, which is not in tension with the low-energy constraints. One must add to Eqs. (A.9)-(A.11) the corresponding RGEs

$$
\partial_{t} Y_{u}^{2}+\left[Y_{u}^{2},\left(\partial_{t} U_{R}^{\dagger}\right) U_{R}\right]=U_{R}^{\dagger}\left(\partial_{t} M_{\bar{U}}\right) U_{R}
$$

$$
\partial_{t}\left(Y^{R} Y^{R^{\dagger}}\right)+\left[Y^{R} Y^{R \dagger},\left(\partial_{t} U_{R}^{T}\right) U_{R}^{*}\right]=U_{R}^{T}\left(\partial_{t} M_{R}\right) U_{R}^{*},
$$

obtained by rotating the squared matrices defined in the flavor basis,

$M_{\bar{U}}=Y_{U}^{\dagger} Y_{U}, \quad M_{R}=Y_{R} Y_{R}^{\dagger}$.

We present here the one-loop gauge-Yukawa-CKM system of equations for $S_{1}$ in the down-origin basis and 2-family approximation. We only give the equations for the parameters affecting the low-scale phenomenology of the $b \rightarrow c$ anomalies. All other parameters can be considered to be zero and relevant at the UV fixed point.

$$
\begin{aligned}
& \frac{d g_{3}}{d t}=-\frac{41}{6} \frac{g_{3}^{3}}{16 \pi^{2}}-f_{g} g_{3} \\
& \frac{d g_{2}}{d t}=-\frac{19}{6} \frac{g_{2}^{3}}{16 \pi^{2}}-f_{g} g_{2} \\
& \frac{d g_{Y}}{d t}= \frac{125}{18} \frac{g_{Y}^{3}}{16 \pi^{2}}-f_{g} g_{Y} \\
& \frac{d y_{c}}{d t}= \frac{1}{16 \pi^{2}}\left\{\left[3 y_{b}^{2}+\frac{9}{2} y_{c}^{2}+3 y_{t}^{2}+y_{\tau}^{2}\right.\right. \\
&-\frac{17}{12} g_{Y}^{2}-\frac{9}{4} g_{2}^{2}-8 g_{3}^{2}-\frac{3}{2} V_{23}^{2} y_{b}^{2}+\frac{1}{2}\left(Y_{23}^{R}\right)^{2} \\
&+\frac{1}{2}\left(V_{22}^{2}\left(\hat{Y}_{23}^{L}\right)^{2}+V_{23}^{2}\left(\hat{Y}_{33}^{L}\right)^{2}\right. \\
&\left.\left.+2 V_{22} V_{23} \hat{Y}_{23}^{L} \hat{Y}_{33}^{L}\right)\right] y_{c} \\
&\left.-2 y_{\tau} Y_{23}^{R}\left(V_{22} \hat{Y}_{23}^{L}+V_{23} \hat{Y}_{33}^{L}\right)\right\}-f_{y} y_{c} \\
& \frac{d y_{t}}{d t}= \frac{1}{16 \pi^{2}}\left\{\left[3 y_{b}^{2}+3 y_{c}^{2}+\frac{9}{2} y_{t}^{2}+y_{\tau}^{2}\right.\right. \\
&-\frac{17}{12} g_{Y}^{2}-\frac{9}{4} g_{2}^{2}-8 g_{3}^{2}-\frac{3}{2} V_{33}^{2} y_{b}^{2}+\frac{1}{2}\left(Y_{33}^{R}\right)^{2} \\
&+\frac{1}{2}\left(V_{32}^{2}\left(\hat{Y}_{23}^{L}\right)^{2}+V_{33}^{2}\left(\hat{Y}_{33}^{L}\right)^{2}\right. \\
&\left.\left.+2 V_{32} V_{33} \hat{Y}_{23}^{L} \hat{Y}_{33}^{L}\right)\right] y_{t} \\
& \frac{d y_{\tau}}{d t}= \frac{1}{16 \pi^{2}}\left\{\left[3 y_{b}^{2}+3 y_{c}^{2}+3 y_{t}^{2}\right.\right. \\
&+\frac{5}{2} y_{\tau}^{2}-\frac{15}{4} g_{Y}^{2}-\frac{9}{4} g_{2}^{2} \\
&+\frac{1}{2}\left(\hat{Y}_{33}^{L} Y_{33}^{R}\left(V_{32} \hat{Y}_{23}^{L}+V_{33} \hat{Y}_{33}^{L}\right)\right\}-f_{y} y_{t} \\
& \frac{1}{16 \pi^{2}}\left\{\left[\frac{9}{2} y_{b}^{2}+3 y_{c}^{2}+3 y_{t}^{2}+y_{\tau}^{2}\right.\right. \\
&-\frac{5}{12} g_{Y}^{2}-\frac{9}{4} g_{2}^{2}-8 g_{3}^{2} \\
&-\frac{3}{2}\left(V_{23}^{2} y_{c}^{2}+V_{33}^{2} y_{t}^{2}\right) \\
& y_{y} y_{b} \\
& \hline
\end{aligned}
$$




$$
\begin{aligned}
& \left.+\frac{3}{2}\left(\left(\hat{Y}_{23}^{L}\right)^{2}+\left(\hat{Y}_{33}^{L}\right)^{2}\right)+\frac{3}{2}\left(\left(Y_{23}^{R}\right)^{2}+\left(Y_{33}^{R}\right)^{2}\right)\right] y_{\tau} \\
& -6\left[y_{c} Y_{23}^{R}\left(V_{22} \hat{Y}_{23}^{L}+V_{23} \hat{Y}_{33}^{L}\right)\right. \\
& \left.\left.+y_{t} Y_{33}^{R}\left(V_{32} \hat{Y}_{23}^{L}+V_{33} \hat{Y}_{33}^{L}\right)\right]\right\}-f_{y} y_{\tau} \\
& \frac{d \hat{Y}_{23}^{L}}{d t}=\frac{1}{16 \pi^{2}}\left\{\left[4\left(\hat{Y}_{23}^{L}\right)^{2}+\frac{7}{2}\left(\hat{Y}_{33}^{L}\right)^{2}+\left(Y_{23}^{R}\right)^{2}\right.\right. \\
& +\left(Y_{33}^{R}\right)^{2}-\frac{5}{6} g_{Y}^{2}-\frac{9}{2} g_{2}^{2}-4 g_{3}^{2}+\frac{1}{2} y_{c}^{2} V_{22}^{2} \\
& +\frac{1}{2} y_{t}^{2} V_{32}^{2} \\
& \left.+\frac{1}{2} y_{\tau}^{2}\right] \hat{Y}_{23}^{L}+2 \hat{Y}_{33}^{L}\left(y_{c}^{2} V_{22} V_{23}\right. \\
& \left.+y_{t}^{2} V_{32} V_{33}\right)-2 y_{\tau}\left(y_{c} V_{22} Y_{23}^{R}\right. \\
& \left.\left.+y_{t} V_{32} Y_{33}^{R}\right)\right\}-f_{y} \hat{Y}_{23}^{L} \\
& \frac{d \hat{Y}_{33}^{L}}{d t}=\frac{1}{16 \pi^{2}}\left\{\left[\frac{9}{2}\left(\hat{Y}_{23}^{L}\right)^{2}+4\left(\hat{Y}_{33}^{L}\right)^{2}\right.\right. \\
& +\left(Y_{23}^{R}\right)^{2}+\left(Y_{33}^{R}\right)^{2}-\frac{5}{6} g_{Y}^{2} \\
& -\frac{9}{2} g_{2}^{2}-4 g_{3}^{2}+\frac{1}{2} y_{b}^{2}+\frac{1}{2} y_{c}^{2} V_{23}^{2} \\
& \left.+\frac{1}{2} y_{t}^{2} V_{33}^{2}+\frac{1}{2} y_{\tau}^{2}\right] \hat{Y}_{33}^{L} \\
& -\hat{Y}_{23}^{L}\left(y_{c}^{2} V_{22} V_{23}+y_{t}^{2} V_{32} V_{33}\right) \\
& \left.-2 y_{\tau}\left(y_{c} V_{23} Y_{23}^{R}+y_{t} V_{33} Y_{33}^{R}\right)\right\}-f_{y} \hat{Y}_{33}^{L} \\
& \frac{d Y_{23}^{R}}{d t}=\frac{1}{16 \pi^{2}}\left\{\left[2\left(\hat{Y}_{23}^{L}\right)^{2}+2\left(\hat{Y}_{33}^{L}\right)^{2}+3\left(Y_{23}^{R}\right)^{2}+3\left(Y_{33}^{R}\right)^{2}\right.\right. \\
& -\frac{1}{2} \frac{y_{t}^{2}+y_{c}^{2}}{y_{t}^{2}-y_{c}^{2}}\left(Y_{33}^{R}\right)^{2}-\frac{13}{3} g_{Y}^{2}-4 g_{3}^{2}+y_{c}^{2}+y_{\tau}^{2} \\
& \left.+\frac{2 y_{t} y_{\tau}}{y_{t}^{2}-y_{c}^{2}}\left(\hat{Y}_{23}^{L} V_{32}+\hat{Y}_{33}^{L} V_{33}\right) Y_{33}^{R}\right] Y_{23}^{R} \\
& -4 y_{c} y_{\tau}\left(V_{22} \hat{Y}_{23}^{L}+V_{23} \hat{Y}_{33}^{L}\right) \\
& +\frac{3 y_{c} y_{t}}{y_{t}^{2}-y_{c}^{2}} y_{b}^{2} V_{23} V_{33} Y_{33}^{R} \\
& +\frac{2 y_{c} y_{\tau}}{y_{t}^{2}-y_{c}^{2}}\left(Y_{33}^{R}\right)^{2}\left(\hat{Y}_{23}^{L} V_{22}+\hat{Y}_{33}^{L} V_{23}\right) \\
& -\frac{y_{c} y_{t}}{y_{t}^{2}-y_{c}^{2}}\left[\left(\hat{Y}_{23}^{L}\right)^{2} V_{22} V_{32}+\left(\hat{Y}_{33}^{L}\right)^{2} V_{23} V_{33}\right. \\
& \left.\left.+\hat{Y}_{23}^{L} \hat{Y}_{33}^{L}\left(V_{23} V_{32}+V_{22} V_{33}\right)\right] Y_{33}^{R}\right\}-f_{y} Y_{23}^{R} \\
& \frac{d Y_{33}^{R}}{d t}=\frac{1}{16 \pi^{2}}\left\{\left[2\left(\hat{Y}_{23}^{L}\right)^{2}+2\left(\hat{Y}_{33}^{L}\right)^{2}\right.\right. \\
& +3\left(Y_{23}^{R}\right)^{2}+\frac{1}{2} \frac{y_{t}^{2}+y_{c}^{2}}{y_{t}^{2}-y_{c}^{2}}\left(Y_{23}^{R}\right)^{2}
\end{aligned}
$$

$$
\begin{aligned}
&+3\left(Y_{33}^{R}\right)^{2}-\frac{13}{3} g_{Y}^{2}-4 g_{3}^{2}+y_{c}^{2}+y_{\tau}^{2} \\
&\left.-\frac{2 y_{c} y_{\tau}}{y_{t}^{2}-y_{c}^{2}}\left(\hat{Y}_{23}^{L} V_{22}+\hat{Y}_{33}^{L} V_{23}\right) Y_{23}^{R}\right] Y_{33}^{R} \\
&-4 y_{t} y_{\tau}\left(V_{32} \hat{Y}_{23}^{L}+V_{33} \hat{Y}_{33}^{L}\right) \\
&-\frac{3 y_{c} y_{t}}{y_{t}^{2}-y_{c}^{2}} y_{b}^{2} V_{23} V_{33} Y_{23}^{R} \\
&-\frac{2 y_{t} y_{\tau}}{y_{t}^{2}-y_{c}^{2}}\left(Y_{23}^{R}\right)^{2}\left(\hat{Y}_{23}^{L} V_{32}+\hat{Y}_{33}^{L} V_{33}\right) \\
&+\frac{y_{c} y_{t}}{y_{t}^{2}-y_{c}^{2}}\left(\left(\hat{Y}_{23}^{L}\right)^{2} V_{22} V_{32}\right. \\
&+\left(\hat{Y}_{33}^{L}\right)^{2} V_{23} V_{33}+\hat{Y}_{23}^{L} \hat{Y}_{33}^{L}\left(V_{23} V_{32}\right. \\
&\left.\left.\left.+V_{22} V_{33}\right)\right) Y_{23}^{R}\right\}-f_{y} Y_{33}^{R} \\
& \frac{d\left|V_{33}\right|}{d t}= \frac{V_{23}}{16 \pi^{2}}\left[-\frac{3}{2} \frac{y_{c}^{2}+y_{t}^{2}}{y_{t}^{2}-y_{c}^{2}} V_{33} V_{23} y_{b}^{2}\right. \\
&+\frac{1}{2} \frac{2 y_{t} y_{c}}{y_{t}^{2}-y_{c}^{2}} Y_{33}^{R} Y_{23}^{R} \\
&+\frac{1}{2} \frac{y_{c}^{2}+y_{t}^{2}}{y_{t}^{2}-y_{c}^{2}}\left(V_{22} V_{32}\left(\hat{Y}_{23}^{L}\right)^{2}+V_{22} V_{33} \hat{Y}_{23}^{L} \hat{Y}_{33}^{L}\right. \\
&\left.+V_{23} V_{32} \hat{Y}_{23}^{L} \hat{Y}_{33}^{L}+V_{23} V_{33}\left(\hat{Y}_{33}^{L}\right)^{2}\right) \\
&-\frac{2 y_{\tau} y_{t}}{y_{t}^{2}-y_{c}^{2}}\left(\hat{Y}_{23}^{L} V_{22}+\hat{Y}_{33}^{L} V_{23}\right) Y_{33}^{R} \\
&\left.-\frac{2 y_{\tau} y_{c}}{y_{t}^{2}-y_{c}^{2}}\left(\hat{Y}_{23}^{L} V_{32}+\hat{Y}_{33}^{L} V_{33}\right) Y_{23}^{R}\right] \\
&-\frac{V_{32}}{16 \pi^{2}}\left[\frac{3}{2}\left(y_{t}^{2} V_{32} V_{33}+y_{c}^{2} V_{22} V_{23}\right)-\frac{1}{2} \hat{Y}_{23}^{L} \hat{Y}_{33}^{L}\right] . \\
& \hline
\end{aligned}
$$

As before, the orthogonality of the CKM matrix in the 2-family approximation leads to

$V_{22}=V_{33}, \quad V_{23}=-V_{32}=\sqrt{1-V_{33^{2}}}$.

\section{References}

1. S. Weinberg, General relativity, in ed. by S.W. Hawking, W. Israel (Cambridge University Press, 1980), pp. 790-831

2. M. Reuter, Nonperturbative evolution equation for quantum gravity. Phys. Rev. D 57, 971-985 (1998). https://doi.org/10.1103/ PhysRevD.57.971. arXiv:hep-th/9605030

3. O. Lauscher, M. Reuter, Ultraviolet fixed point and generalized flow equation of quantum gravity. Phys. Rev. D 65, 025013 (2002). https://doi.org/10.1103/PhysRevD.65.025013. arXiv:hep-th/0108040

4. M. Reuter, F. Saueressig, Renormalization group flow of quantum gravity in the Einstein-Hilbert truncation. Phys. Rev. D 65 , 065016 (2002). https://doi.org/10.1103/PhysRevD.65.065016. arXiv:hep-th/0110054

5. O. Lauscher, M. Reuter, Flow equation of quantum Einstein gravity in a higher derivative truncation. Phys. Rev. D 66, 
025026 (2002). https://doi.org/10.1103/PhysRevD.66.025026. arXiv:hep-th/0205062

6. D.F. Litim, Fixed points of quantum gravity. Phys. Rev. Lett. 92, 201301 (2004). https://doi.org/10.1103/PhysRevLett.92.201301. arXiv:hep-th/0312114

7. A. Codello, R. Percacci, Fixed points of higher derivative gravity. Phys. Rev. Lett. 97, 221301 (2006). https://doi.org/10.1103/ PhysRevLett.97.221301. arXiv:hep-th/0607128

8. P.F. Machado, F. Saueressig, On the renormalization group flow of $\mathrm{f}(\mathrm{R})$-gravity. Phys. Rev. D 77, 124045 (2008). https://doi.org/ 10.1103/PhysRevD.77.124045. arXiv:0712.0445 [hep-th]

9. A. Codello, R. Percacci, C. Rahmede, Investigating the ultraviolet properties of gravity with a Wilsonian renormalization group equation. Ann. Phys. 324, 414-469 (2009). https://doi.org/10. 1016/j.aop.2008.08.008. arXiv:0805.2909 [hep-th]

10. D. Benedetti, P.F. Machado, F. Saueressig, Asymptotic safety in higher-derivative gravity. Mod. Phys. Lett. A 24, 22332241 (2009). https://doi.org/10.1142/S0217732309031521. arXiv:0901.2984 [hep-th]

11. E. Manrique, S. Rechenberger, F. Saueressig, Asymptotically safe Lorentzian gravity. Phys. Rev. Lett. 106, 251302 (2011). https:// doi.org/10.1103/PhysRevLett.106.251302. arXiv:1102.5012 [hep-th]

12. J.A. Dietz, T.R. Morris, Asymptotic safety in the $f(R)$ approximation. JHEP 01, 108 (2013). https://doi.org/10.1007/ JHEP01(2013)108. arXiv:1211.0955 [hep-th]

13. K. Falls, D. Litim, K. Nikolakopoulos, C. Rahmede, A bootstrap towards asymptotic safety, arXiv:1301.4191 [hep-th]

14. K. Falls, D.F. Litim, K. Nikolakopoulos, C. Rahmede, Further evidence for asymptotic safety of quantum gravity. Phys. Rev. D 93(10), 104022104022 (2016). https://doi.org/10.1103/ PhysRevD.93.104022. arXiv:1410.4815 [hep-th]

15. S.P. Robinson, F. Wilczek, Gravitational correction to running of gauge couplings. Phys. Rev. Lett. 96, 231601 (2006). https://doi. org/10.1103/PhysRevLett.96.231601. arXiv:hep-th/0509050

16. A.R. Pietrykowski, Gauge dependence of gravitational correction to running of gauge couplings. Phys. Rev. Lett. 98, 061801 (2007). https://doi.org/10.1103/PhysRevLett.98.061801. arXiv:hep-th/0606208

17. D.J. Toms, Quantum gravity and charge renormalization. Phys. Rev. D 76, 045015 (2007). https://doi.org/10.1103/PhysRevD.76. 045015. arXiv:0708.2990 [hep-th]

18. Y. Tang, Y.-L. Wu, Gravitational contributions to the running of gauge couplings. Commun. Theor. Phys. 54, 1040-1044 (2010). https://doi.org/10.1088/0253-6102/54/6/15. arXiv:0807.0331 [hep-ph]

19. D.J. Toms, Cosmological constant and quantum gravitational corrections to the running fine structure constant. Phys. Rev. Lett. 101, 131301 (2008). https://doi.org/10.1103/PhysRevLett.101. 131301. arXiv:0809.3897 [hep-th]

20. A. Rodigast, T. Schuster, Gravitational corrections to Yukawa and phi**4 interactions. Phys. Rev. Lett. 104, 081301 (2010). https:// doi.org/10.1103/PhysRevLett.104.081301. arXiv:0908.2422 [hep-th]

21. O. Zanusso, L. Zambelli, G. Vacca, R. Percacci, Gravitational corrections to Yukawa systems. Phys. Lett. B 689, 90-94 (2010). https://doi.org/10.1016/j.physletb.2010.04.043. arXiv:0904.0938 [hep-th]

22. J.-E. Daum, U. Harst, M. Reuter, Running gauge coupling in asymptotically safe quantum gravity. JHEP 01, 084 (2010). https://doi.org/10.1007/JHEP01(2010)084. arXiv:0910.4938 [hep-th]

23. J.-E. Daum, U. Harst, M. Reuter, Non-perturbative QEG corrections to the Yang-Mills beta function. Gen. Relativ. Gravit. 43, 2393 (2011). https://doi.org/10.1007/s10714-010-1032-2. arXiv: 1005.1488 [hep-th]
24. S. Folkerts, D.F. Litim, J.M. Pawlowski, Asymptotic freedom of Yang-Mills theory with gravity. Phys. Lett. B 709, 234-241 (2012). https://doi.org/10.1016/j.physletb.2012.02.002. arXiv:1101.5552 [hep-th]

25. K.-Y. Oda, M. Yamada, Non-minimal coupling in Higgs-Yukawa model with asymptotically safe gravity. Class. Quantum Gravity 33(12), 125011 (2016). https://doi.org/10.1088/0264-9381/ 33/12/125011. arXiv:1510.03734 [hep-th]

26. A. Eichhorn, A. Held, J.M. Pawlowski, Quantum-gravity effects on a Higgs-Yukawa model. Phys. Rev. D 94(10), 104027 (2016). https://doi.org/10.1103/PhysRevD.94.104027. arXiv:1604.02041 [hep-th]

27. N. Christiansen, A. Eichhorn, An asymptotically safe solution to the $\mathrm{U}(1)$ triviality problem. Phys. Lett. B 770, 154-160 (2017). https://doi.org/10.1016/j.physletb.2017.04.047. arXiv: 1702.07724 [hep-th]

28. Y. Hamada, M. Yamada, Asymptotic safety of higher derivative quantum gravity non-minimally coupled with a matter system. JHEP 08, 070 (2017). https://doi.org/10.1007/JHEP08(2017)070. arXiv: 1703.09033 [hep-th]

29. N. Christiansen, D.F. Litim, J.M. Pawlowski, M. Reichert, Asymptotic safety of gravity with matter. Phys. Rev. D 97(10), 106012 (2018). https://doi.org/10.1103/PhysRevD.97.106012. arXiv: 1710.04669 [hep-th]

30. A. Eichhorn, A. Held, Viability of quantum-gravity induced ultraviolet completions for matter. Phys. Rev. D 96(8), 086025 (2017). https://doi.org/10.1103/PhysRevD.96.086025. arXiv: 1705.02342 [gr-qc]

31. C. Wetterich, Exact evolution equation for the effective potential. Phys. Lett. B 301(1), 90-94 (1993). https://doi.org/10.1016/ 0370-2693(93)90726-X

32. U. Harst, M. Reuter, QED coupled to QEG. JHEP 05, 119 (2011). https://doi.org/10.1007/JHEP05(2011)119. arXiv:1101.6007 [hep-th]

33. A. Eichhorn, F. Versteegen, Upper bound on the Abelian gauge coupling from asymptotic safety. JHEP 01, 030 (2018). https:// doi.org/10.1007/JHEP01(2018)030. arXiv:1709.07252 [hep-th]

34. A. Eichhorn, A. Held, Top mass from asymptotic safety. Phys. Lett. B 777, 217-221 (2018). https://doi.org/10.1016/j.physletb. 2017.12.040. arXiv:1707.01107 [hep-th]

35. A. Eichhorn, A. Held, Mass difference for charged quarks from asymptotically safe quantum gravity. Phys. Rev. Lett. 121(15), 151302151302 (2018). https://doi.org/10.1103/PhysRevLett. 121.151302. arXiv: 1803.04027 [hep-th]

36. M. Shaposhnikov, C. Wetterich, Asymptotic safety of gravity and the Higgs boson mass. Phys. Lett. B 683, 196-200 (2010). https:// doi.org/10.1016/j.physletb.2009.12.022. arXiv:0912.0208 [hepth]

37. R. Alkofer, A. Eichhorn, A. Held, C.M. Nieto, R. Percacci, M. Schröfl, Quark masses and mixings in minimally parameterized UV completions of the Standard Model. arXiv:2003.08401 [hep-ph]

38. Z.-W. Wang, F.S. Sage, T.G. Steele, R.B. Mann, Asymptotic safety in the conformal hidden sector? J. Phys. G 45(9), 095002 (2018). https://doi.org/10.1088/1361-6471/ aad2c7. arXiv:1511.02531 [hep-ph]

39. F. Grabowski, J.H. Kwapisz, K.A. Meissner, Asymptotic safety and conformal standard model. Phys. Rev. D 99(11), 115029 (2019). https://doi.org/10.1103/PhysRevD.99.115029. arXiv:1810.08461 [hep-ph]

40. J.H. Kwapisz, Asymptotic safety, the Higgs boson mass, and beyond the standard model physics. Phys. Rev. D 100(11), 115001115001 (2019). https://doi.org/10.1103/PhysRevD.100. 115001. arXiv:1907.12521 [hep-ph] 
41. M. Reichert, J. Smirnov, Dark matter meets quantum gravity. Phys. Rev. D 101(6), 063015 (2020). https://doi.org/10.1103/ PhysRevD.101.063015. arXiv:1911.00012 [hep-ph]

42. A. Eichhorn, M. Pauly, Safety in darkness: Higgs portal to simple Yukawa systems. arXiv:2005.03661 [hep-ph]

43. LHCb Collaboration, R. Aaij et al., Test of lepton universality using $B^{+} \rightarrow K^{+} \ell^{+} \ell^{-}$decays. Phys. Rev. Lett. 113, 151601 (2014). https://doi.org/10.1103/PhysRevLett.113. 151601. arXiv:1406.6482 [hep-ex]

44. LHCb Collaboration, R. Aaij et al., Differential branching fractions and isospin asymmetries of $B \rightarrow K^{(*)} \mu^{+} \mu^{-}$decays. JHEP 06, 133 (2014). https://doi.org/10.1007/JHEP06(2014)133. arXiv: 1403.8044 [hep-ex]

45. LHCb Collaboration, R. Aaij et al., Angular analysis of the $B^{0} \rightarrow K^{* 0} \mu^{+} \mu^{-}$decay using $3 \mathrm{fb}^{-1}$ of integrated luminosity. JHEP 02, 104 (2016). https://doi.org/10.1007/JHEP02(2016)104. arXiv: 1512.04442 [hep-ex]

46. LHCb Collaboration, R. Aaij et al., Angular analysis and differential branching fraction of the decay $B_{s}^{0} \rightarrow \phi \mu^{+} \mu^{-}$. JHEP 09, 179 (2015). https://doi.org/10.1007/JHEP09(2015)179. arXiv:1506.08777 [hep-ex]

47. LHCb Collaboration, R. Aaij et al., Measurements of the S-wave fraction in $B^{0} \rightarrow K^{+} \pi^{-} \mu^{+} \mu^{-}$decays and the $B^{0} \rightarrow K^{*}(892)^{0} \mu^{+} \mu^{-}$differential branching fraction. JHEP 11, 047 (2016). https://doi.org/10.1007/JHEP11(2016)047. arXiv: 1606.04731 [hep-ex] (Erratum: JHEP 04, 142 (2017))

48. LHCb Collaboration, R. Aaij et al., Test of lepton universality with $B^{0} \rightarrow K^{* 0} \ell^{+} \ell^{-}$decays. JHEP 08, 055 (2017). https://doi. org/10.1007/JHEP08(2017)055. arXiv:1705.05802 [hep-ex]

49. LHCb Collaboration, R. Aaij et al., Search for lepton-universality violation in $B^{+} \rightarrow K^{+} \ell^{+} \ell^{-}$decays. Phys. Rev. Lett. 122(19), 191801 (2019). https://doi.org/10.1103/PhysRevLett. 122.191801. arXiv: 1903.09252 [hep-ex]

50. LHCb Collaboration, R. Aaij et al., Measurement of $C P$-averaged observables in the $B^{0} \rightarrow K^{* 0} \mu^{+} \mu^{-}$decay. arXiv:2003.04831 [hep-ex]

51. Belle Collaboration, S. Wehle et al., Lepton-flavor-dependent angular analysis of $B \rightarrow K^{*} \ell^{+} \ell^{-}$. Phys. Rev. Lett. 118(11), 111801 (2017). https://doi.org/10.1103/PhysRevLett. 118.111801. arXiv:1612.05014 [hep-ex]

52. Belle Collaboration, A. Abdesselam et al., Test of lepton flavor universality in $B \rightarrow K^{*} \ell^{+} \ell^{-}$decays at Belle. arXiv:1904.02440 [hep-ex]

53. C.M.S. Collaboration, A.M. Sirunyan et al., Measurement of angular parameters from the decay $\mathrm{B}^{0} \rightarrow \mathrm{K}^{* 0} \mu^{+} \mu^{-}$in proton-proton collisions at $\sqrt{s}=8 \mathrm{TeV}$. Phys. Lett. B 781, 517-541 (2018). https://doi.org/10.1016/j.physletb.2018.04.030. arXiv: 1710.02846 [hep-ex]

54. ATLAS Collaboration, M. Aaboud et al., Angular analysis of $B_{d}^{0} \rightarrow K^{*} \mu^{+} \mu^{-}$decays in $p p$ collisions at $\sqrt{s}=8 \mathrm{TeV}$ with the ATLAS detector. JHEP 10, 047 (2018). https://doi.org/10.1007/ JHEP10(2018)047. arXiv:1805.04000 [hep-ex]

55. BaBar Collaboration, J. Lees et al., Evidence for an excess of $\bar{B} \rightarrow D^{(*)} \tau^{-} \bar{v}_{\tau}$ decays. Phys. Rev. Lett. 109, 101802 (2012). https://doi.org/10.1103/PhysRevLett.109. 101802. arXiv:1205.5442 [hep-ex]

56. BaBar Collaboration, J. Lees et al., Measurement of an Excess of $\bar{B} \rightarrow D^{(*)} \tau^{-} \bar{\nu}_{\tau}$ decays and implications for charged Higgs bosons. Phys. Rev. D 88(7), 072012 (2013). https://doi.org/10. 1103/PhysRevD.88.072012. arXiv:1303.0571 [hep-ex]

57. Belle Collaboration, M. Huschle et al., Measurement of the branching ratio of $\bar{B} \rightarrow D^{(*)} \tau^{-} \bar{\nu}_{\tau}$ relative to $\bar{B} \rightarrow D^{(*)} \ell^{-} \bar{\nu}_{\ell}$ decays with hadronic tagging at Belle. Phys. Rev. D 92(7), 072014 (2015). https://doi.org/10.1103/PhysRevD.92.072014. arXiv:1507.03233 [hep-ex]
58. Belle Collaboration, Y. Sato et al., Measurement of the branching ratio of $\bar{B}^{0} \rightarrow D^{*+} \tau^{-} \bar{v}_{\tau}$ relative to $\bar{B}^{0} \rightarrow D^{*+} \ell^{-} \bar{v}_{\ell}$ decays with a semileptonic tagging method. Phys. Rev. D 94(7), 072007 (2016). https://doi.org/10.1103/PhysRevD.94.072007. arXiv:1607.07923 [hep-ex]

59. Belle Collaboration, S. Hirose et al., Measurement of the $\tau$ lepton polarization and $R\left(D^{*}\right)$ in the decay $\bar{B} \rightarrow D^{*} \tau^{-} \bar{v}_{\tau}$. Phys. Rev. Lett. 118(21), 211801 (2017). https://doi.org/10.1103/ PhysRevLett.118.211801. arXiv:1612.00529 [hep-ex]

60. LHCb Collaboration, R. Aaij et al., Measurement of the ratio of branching fractions $\mathscr{B}\left(\bar{B}^{0} \rightarrow D^{*+} \tau^{-} \bar{\nu}_{\tau}\right) / \mathscr{B}\left(\bar{B}^{0} \rightarrow\right.$ $\left.D^{*+} \mu^{-} \bar{v}_{\mu}\right)$. Phys. Rev. Lett. 115(11), 111803 (2015). https://doi. org/10.1103/PhysRevLett.115.111803. arXiv:1506.08614 [hepex] (Erratum: Phys. Rev. Lett. 115, 159901 (2015))

61. LHCb Collaboration, R. Aaij et al., Measurement of the ratio of the $B^{0} \rightarrow D^{*-} \tau^{+} v_{\tau}$ and $B^{0} \rightarrow D^{*-} \mu^{+} v_{\mu}$ branching fractions using three-prong $\tau$-lepton decays. Phys. Rev. Lett. 120(17), 171802 (2018). https://doi.org/10.1103/PhysRevLett. 120.171802. arXiv:1708.08856 [hep-ex]

62. LHCb Collaboration, R. Aaij et al., Test of Lepton Flavor Universality by the measurement of the $B^{0} \rightarrow D^{*-} \tau^{+} v_{\tau}$ branching fraction using three-prong $\tau$ decays. Phys. Rev. D 97(7), 072013 (2018). https://doi.org/10.1103/PhysRevD.97. 072013. arXiv:1711.02505 [hep-ex]

63. Belle Collaboration, A. Abdesselam et al., Measurement of $\mathscr{R}(D)$ and $\mathscr{R}\left(D^{*}\right)$ with a semileptonic tagging method. arXiv:1904.08794 [hep-ex]

64. Belle Collaboration, G. Caria et al., Measurement of $\mathscr{R}(D)$ and $\mathscr{R}\left(D^{*}\right)$ with a semileptonic tagging method. Phys. Rev. Lett. 124(16), 161803 (2020). https://doi.org/10.1103/PhysRevLett. 124.161803. arXiv: 1910.05864 [hep-ex]

65. I. Doršner, S. Fajfer, A. Greljo, J. Kamenik, N. Košnik, Physics of leptoquarks in precision experiments and at particle colliders. Phys. Rep. 641, 1-68 (2016). https://doi.org/10.1016/j.physrep. 2016.06.001. arXiv:1603.04993 [hep-ph]

66. P. Donà, A. Eichhorn, R. Percacci, Matter matters in asymptotically safe quantum gravity. Phys. Rev. D 89(8), 084035 (2014). https://doi.org/10.1103/PhysRevD.89.084035. arXiv:1311.2898 [hep-th]

67. D.F. Litim, Renormalisation group and the Planck scale. Philos. Trans. R. Soc. Lond. A 369, 2759-2778 (2011). https://doi.org/ 10.1098/rsta.2011.0103. arXiv:1102.4624 [hep-th]

68. A.D. Bond, D.F. Litim, Theorems for asymptotic safety of gauge theories. Eur. Phys. J. C 77(6), 429 (2017). https://doi. org/10.1140/epjc/s10052-017-4976-5. arXiv:1608.00519 [hepth] (Erratum: Eur. Phys. J. C 77, 525 (2017))

69. A.D. Bond, D.F. Litim, Price of asymptotic safety. Phys. Rev. Lett. 122(21), 211601 (2019). https://doi.org/10.1103/PhysRevLett. 122.211601. arXiv:1801.08527 [hep-th]

70. A. Codello, R. Percacci, C. Rahmede, Ultraviolet properties of f(R)-gravity. Int. J. Mod. Phys. A 23, 143-150 (2008). https://doi. org/10.1142/S0217751X08038135. arXiv:0705.1769 [hep-th]

71. K. Falls, C.R. King, D.F. Litim, K. Nikolakopoulos, C. Rahmede, Asymptotic safety of quantum gravity beyond Ricci scalars. Phys. Rev. D 97(8), 086006 (2018). https://doi.org/10.1103/PhysRevD. 97.086006. arXiv:1801.00162 [hep-th]

72. K.G. Falls, D.F. Litim, J. Schröder, Aspects of asymptotic safety for quantum gravity. Phys. Rev. D 99(12), 126015 (2019). https:// doi.org/10.1103/PhysRevD.99.126015. arXiv:1810.08550 [grqc]

73. G. Narain, R. Percacci, On the scheme dependence of gravitational beta functions. Acta Phys. Pol. B 40, 3439-3457 (2009). arXiv:0910.5390 [hep-th]

74. W. Altmannshofer, D.M. Straub, New physics in $b \rightarrow$ $s$ transitions after LHC run 1. Eur. Phys. J. C 75(8), 
382 (2015). https://doi.org/10.1140/epjc/s10052-015-3602-7. arXiv: 1411.3161 [hep-ph]

75. W. Altmannshofer, C. Niehoff, P. Stangl, D.M. Straub, Status of the $B \rightarrow K^{*} \mu^{+} \mu^{-}$anomaly after Moriond 2017. Eur. Phys. J. C 77(6), 377 (2017). https://doi.org/10.1140/epjc/ s10052-017-4952-0. arXiv:1703.09189 [hep-ph]

76. B. Capdevila, A. Crivellin, S. Descotes-Genon, J. Matias, J. Virto, Patterns of new physics in $b \rightarrow s \ell^{+} \ell^{-}$transitions in the light of recent data. JHEP 01, 093 (2018). https://doi.org/10.1007/ JHEP01(2018)093. arXiv:1704.05340 [hep-ph]

77. W. Altmannshofer, P. Stangl, D.M. Straub, Interpreting hints for lepton flavor universality violation. Phys. Rev. D 96(5), 055008 (2017). https://doi.org/10.1103/PhysRevD.96.055008. arXiv:1704.05435 [hep-ph]

78. G. D'Amico, M. Nardecchia, P. Panci, F. Sannino, A. Strumia, R. Torre, A. Urbano, Flavour anomalies after the $R_{K^{*}}$ measurement. JHEP 09, 010 (2017). https://doi.org/10.1007/JHEP09(2017)010. arXiv:1704.05438 [hep-ph]

79. M. Ciuchini, A.M. Coutinho, M. Fedele, E. Franco, A. Paul, L. Silvestrini, M. Valli, On flavourful easter eggs for new physics hunger and lepton flavour universality violation. Eur. Phys. J. C 77(10), 688 (2017). https://doi.org/10.1140/epjc/ s10052-017-5270-2. arXiv:1704.05447 [hep-ph]

80. A.K. Alok, B. Bhattacharya, A. Datta, D. Kumar, J. Kumar, D. London, New physics in $b \rightarrow s \mu^{+} \mu^{-}$after the measurement of $R_{K^{*}}$. Phys. Rev. D 96(9), 095009 (2017). https://doi.org/10.1103/ PhysRevD.96.095009. arXiv:1704.07397 [hep-ph]

81. T. Hurth, F. Mahmoudi, S. Neshatpour, Global fits to $b \rightarrow$ sll data and signs for lepton non-universality. JHEP 12, 053 (2014). https://doi.org/10.1007/JHEP12(2014)053. arXiv: 1410.4545 [hep-ph]

82. T. Hurth, F. Mahmoudi, S. Neshatpour, On the anomalies in the latest LHCb data. Nucl. Phys. B 909, 737-777 (2016). https://doi. org/10.1016/j.nuclphysb.2016.05.022. arXiv:1603.00865 [hep$\mathrm{ph}]$

83. V.G. Chobanova, T. Hurth, F. Mahmoudi, D.M. Santos, S. Neshatpour, Large hadronic power corrections or new physics in the rare decay $B \rightarrow K^{*} \mu^{+} \mu^{-}$? JHEP 07, 025 (2017). https://doi.org/10. 1007/JHEP07(2017)025. arXiv:1702.02234 [hep-ph]

84. T. Hurth, F. Mahmoudi, D.M. Santos, S. Neshatpour, Lepton nonuniversality in exclusive $b \rightarrow$ sll decays. Phys. Rev. D 96(9), 095034 (2017). https://doi.org/10.1103/PhysRevD.96. 095034. arXiv:1705.06274 [hep-ph]

85. A. Arbey, T. Hurth, F. Mahmoudi, S. Neshatpour, Hadronic and new physics contributions to $b \rightarrow s$ transitions. Phys. Rev. D 98(9), 095027 (2018). https://doi.org/10.1103/PhysRevD.98. 095027. arXiv:1806.02791 [hep-ph]

86. M. Algueró, B. Capdevila, A. Crivellin, S. Descotes-Genon, P. Masjuan, J. Matias, M. Novoa Brunet, J. Virto, Emerging patterns of new physics with and without lepton flavour universal contributions. Eur. Phys. J. C 79(8), 714 (2019). https://doi. org/10.1140/epjc/s10052-019-7216-3. arXiv:1903.09578 [hepph] (Addendum: Eur. Phys. J. C 80, 511 (2020))

87. A.K. Alok, A. Dighe, S. Gangal, D. Kumar, Continuing search for new physics in $b \rightarrow s \mu \mu$ decays: two operators at a time. JHEP 06, 089 (2019). https://doi.org/10.1007/JHEP06(2019)089. arXiv:1903.09617 [hep-ph]

88. M. Ciuchini, A.M. Coutinho, M. Fedele, E. Franco, A. Paul, L. Silvestrini, M. Valli, New physics in $b \rightarrow s \ell^{+} \ell^{-}$confronts new data on lepton universality. Eur. Phys. J. C 79(8), 719 (2019). https://doi.org/10.1140/epjc/s10052-019-7210-9. arXiv:1903.09632 [hep-ph]

89. A. Datta, J. Kumar, D. London, The $B$ anomalies and new physics in $b \rightarrow s e^{+} e^{-}$. Phys. Lett. B 797, 134858 (2019). https://doi.org/ 10.1016/j.physletb.2019.134858. arXiv:1903.10086 [hep-ph]
90. J. Aebischer, W. Altmannshofer, D. Guadagnoli, M. Reboud, P. Stangl, D.M. Straub, B-decay discrepancies after Moriond 2019. Eur. Phys. J. C 80(3), 252 (2020). https://doi.org/10.1140/epjc/ s10052-020-7817-x. arXiv:1903.10434 [hep-ph]

91. K. Kowalska, D. Kumar, E.M. Sessolo, Implications for new physics in $b \rightarrow s \mu \mu$ transitions after recent measurements by Belle and LHCb. Eur. Phys. J. C 79(10), 840 (2019). https://doi. org/10.1140/epjc/s10052-019-7330-2. arXiv:1903.10932 [hep$\mathrm{ph}]$

92. A. Arbey, T. Hurth, F. Mahmoudi, D.M. Santos, S. Neshatpour, Update on the $\mathrm{b} \rightarrow \mathrm{s}$ anomalies. Phys. Rev. D 100(1), 015045 (2019). https://doi.org/10.1103/PhysRevD.100.015045. arXiv:1904.08399 [hep-ph]

93. S. Bhattacharya, A. Biswas, S. Nandi, S.K. Patra, Exhaustive model selection in $b \rightarrow$ sll decays: pitting cross-validation against the Akaike information criterion. Phys. Rev. D 101(5), 055025 (2020). https://doi.org/10.1103/PhysRevD.101.055025. arXiv:1908.04835 [hep-ph]

94. G. Hiller, D. Loose, K. Schönwald, Leptoquark flavor patterns and B decay anomalies. JHEP 12, 027 (2016). https://doi.org/10. 1007/JHEP12(2016)027. arXiv:1609.08895 [hep-ph]

95. I. Doršner, S. Fajfer, D.A. Faroughy, N. Košnik, The role of the $S_{3}$ GUT leptoquark in flavor universality and collider searches. JHEP 10, 188 (2017). https://doi.org/10.1007/JHEP10(2017)188. arXiv:1706.07779 [hep-ph]

96. A. Crivellin, D. Müller, T. Ota, Simultaneous explanation of $R_{D^{(*)}}$ and $b \rightarrow s \mu^{+} \mu^{-}$: the last scalar leptoquarks standing. JHEP 09, 040 (2017). https://doi.org/10.1007/JHEP09(2017)040. arXiv:1703.09226 [hep-ph]

97. G. Hiller, I. Nisandzic, $R_{K}$ and $R_{K^{*}}$ beyond the standard model. Phys. Rev. D 96(3), 035003 (2017). https://doi.org/10.1103/ PhysRevD.96.035003. arXiv:1704.05444 [hep-ph]

98. D. Buttazzo, A. Greljo, G. Isidori, D. Marzocca, B-physics anomalies: a guide to combined explanations. JHEP 11, 044 (2017). https://doi.org/10.1007/JHEP11(2017)044. arXiv:1706.07808 [hep-ph]

99. G. Hiller, D. Loose, I. Nišandžić, Flavorful leptoquarks at hadron colliders. Phys. Rev. D 97(7), 075004 (2018). https://doi.org/10. 1103/PhysRevD.97.075004. arXiv:1801.09399 [hep-ph]

100. D. Bečirević, I. Doršner, S. Fajfer, N. Košnik, D.A. Faroughy, O. Sumensari, Scalar leptoquarks from grand unified theories to accommodate the $B$-physics anomalies. Phys. Rev. D 98(5), 055003 (2018). https://doi.org/10.1103/PhysRevD.98. 055003. arXiv: 1806.05689 [hep-ph]

101. A. Angelescu, D. Bečirević, D. Faroughy, O. Sumensari, Closing the window on single leptoquark solutions to the $B$ physics anomalies. JHEP 10, 183 (2018). https://doi.org/10.1007/ JHEP10(2018)183. arXiv:1808.08179 [hep-ph]

102. D. Buttazzo, G. Degrassi, P.P. Giardino, G.F. Giudice, F. Sala, A. Salvio, A. Strumia, Investigating the near-criticality of the Higgs boson. JHEP 12, 089 (2013). https://doi.org/10.1007/ JHEP12(2013)089. arXiv:1307.3536 [hep-ph]

103. ATLAS Collaboration, G. Aad et al., Search for pairs of scalar leptoquarks decaying into quarks and electrons or muons in $\sqrt{s}=$ $13 \mathrm{TeV}$ pp collisions with the ATLAS detector. arXiv:2006.05872 [hep-ex]

104. FCC Collaboration, A. Abada et al., FCC physics opportunities: future circular collider conceptual design report volume 1. Eur. Phys. J. C 79(6), 474 (2019). https://doi.org/10.1140/epjc/ s10052-019-6904-3

105. BNL E871 Collaboration, D. Ambrose et al., Improved branching ratio measurement for the decay $K_{L}^{0} \rightarrow \mu^{+} \mu^{-}$. Phys. Rev. Lett. 84, 1389-1392 (2000). https://doi.org/10.1103/PhysRevLett.84. 1389 
106. R. Mandal, A. Pich, Constraints on scalar leptoquarks from lepton and kaon physics. JHEP 12, 089 (2019). https://doi.org/10.1007/ JHEP12(2019)089. arXiv:1908.11155 [hep-ph]

107. LHCb Collaboration, R. Aaij et al., Search for the rare decay $D^{0} \rightarrow \mu^{+} \mu^{-}$. Phys. Lett. B 725, 15-24 (2013). https://doi.org/ 10.1016/j.physletb.2013.06.037. arXiv:1305.5059 [hep-ex]

108. K. Kowalska, E.M. Sessolo, Y. Yamamoto, Constraints on charmphilic solutions to the muon g-2 with leptoquarks. Phys. Rev. D 99(5), 055007 (2019). https://doi.org/10.1103/PhysRevD.99. 055007. arXiv:1812.06851 [hep-ph]

109. Y. Sakaki, M. Tanaka, A. Tayduganov, R. Watanabe, Testing leptoquark models in $\bar{B} \rightarrow D^{(*)} \tau \bar{\nu}$. Phys. Rev. D 88(9), 094012 (2013). https://doi.org/10.1103/PhysRevD.88.094012. arXiv:1309.0301 [hep-ph]

110. S. Fajfer, N. Košnik, Vector leptoquark resolution of $R_{K}$ and $R_{D^{(*)}}$ puzzles. Phys. Lett. B 755, 270-274 (2016). https://doi.org/10. 1016/j.physletb.2016.02.018. arXiv:1511.06024 [hep-ph]

111. D. Bečirević, S. Fajfer, N. Košnik, O. Sumensari, Leptoquark model to explain the $B$-physics anomalies, $R_{K}$ and $R_{D}$. Phys. Rev. D 94(11), 115021 (2016). https://doi.org/10.1103/PhysRevD.94. 115021. arXiv:1608.08501 [hep-ph]

112. X.-Q. Li, Y.-D. Yang, X. Zhang, Revisiting the one leptoquark solution to the $R_{D^{(*)}}$ anomalies and its phenomenological implications. JHEP 08, 054 (2016). https://doi.org/10.1007/ JHEP08(2016)054. arXiv:1605.09308 [hep-ph]

113. D. Marzocca, Addressing the B-physics anomalies in a fundamental composite Higgs model. JHEP 07, 121 (2018). https://doi.org/ 10.1007/JHEP07(2018)121. arXiv:1803.10972 [hep-ph]

114. S. Iguro, T. Kitahara, Y. Omura, R. Watanabe, K. Yamamoto, D* polarization vs. $R_{D^{(*)}}$ anomalies in the leptoquark models. JHEP 02, 194 (2019). https://doi.org/10.1007/JHEP02(2019)194. arXiv:1811.08899 [hep-ph]

115. S. Bansal, R.M. Capdevilla, C. Kolda, Constraining the minimal flavor violating leptoquark explanation of the $R_{D^{(*)}}$ anomaly. Phys. Rev. D 99(3), 035047 (2019). https://doi.org/10.1103/ PhysRevD.99.035047. arXiv:1810.11588 [hep-ph]

116. H. Yan, Y.-D. Yang, X.-B. Yuan, Phenomenology of $b \rightarrow c \tau \bar{v}$ decays in a scalar leptoquark model. Chin. Phys. C 43(8), 083105083105 (2019). https://doi.org/10.1088/1674-1137/43/8/ 083105. arXiv:1905.01795 [hep-ph]

117. O. Popov, M.A. Schmidt, G. White, $R_{2}$ as a single leptoquark solution to $R_{D^{(*)}}$ and $R_{K^{(*)}}$. Phys. Rev. D 100(3), 035028 (2019). https://doi.org/10.1103/PhysRevD.100.035028. arXiv:1905.06339 [hep-ph]

118. K. Cheung, Z.-R. Huang, H.-D. Li, C.-D. Lü, Y.-N. Mao, R.-Y. Tang, Revisit to the $b \rightarrow c \tau v$ transition: in and beyond the SM. arXiv:2002.07272 [hep-ph]

119. V. Gherardi, D. Marzocca, E. Venturini, Matching scalar leptoquarks to the SMEFT at one loop. JHEP 07, 225 (2020). https:// doi.org/10.1007/JHEP07(2020)225. arXiv:2003.12525 [hep-ph]

120. C. Murgui, A. Peñuelas, M. Jung, A. Pich, Global fit to $b \rightarrow$ $c \tau v$ transitions. JHEP 09, 103 (2019). https://doi.org/10.1007/ JHEP09(2019)103. arXiv:1904.09311 [hep-ph]

121. D. Bardhan, D. Ghosh, $B$-meson charged current anomalies: the post-Moriond 2019 status. Phys. Rev. D 100(1), 011701 (2019). https://doi.org/10.1103/PhysRevD.100.011701. arXiv:1904.10432 [hep-ph]

122. M. Blanke, A. Crivellin, T. Kitahara, M. Moscati, U. Nierste, I. Nišandžić, Addendum to impact of polarization observables and $B_{c} \rightarrow \tau \nu$ on new physics explanations of the $b \rightarrow c \tau \nu$ anomaly. arXiv:1905.08253 [hep-ph]. (Addendum: Phys. Rev. D 100, 035035 (2019))

123. A.K. Alok, D. Kumar, S. Kumbhakar, S.U. Sankar, Solutions to $R_{D^{-}} R_{D^{*}}$ in light of Belle 2019 data. Nucl. Phys. B 953, 114957 (2020). https://doi.org/10.1016/j.nuclphysb.2020. 114957. arXiv:1903.10486 [hep-ph]
124. R.-X. Shi, L.-S. Geng, B. Grinstein, S. Jäger, J. Martin Camalich, Revisiting the new-physics interpretation of the $b \rightarrow c \tau v$ data. JHEP 12, 065 (2019). https://doi.org/10.1007/JHEP12(2019)065. arXiv:1905.08498 [hep-ph]

125. D. Bečirević, M. Fedele, I. Nišandžić, A. Tayduganov, Lepton Flavor Universality tests through angular observables of $\bar{B} \rightarrow$ $D^{(*)} \ell \bar{v}$ decay modes. arXiv:1907.02257 [hep-ph]

126. S.M. Trilov, Search for $K^{+} \rightarrow \pi^{+} \bar{v} v$ at CERN. PoS NuFACT2018 042 (2019). https://doi.org/10.22323/1.341.0042

127. A.J. Buras, J. Girrbach-Noe, C. Niehoff, D.M. Straub, $B \rightarrow K^{(*)} v \bar{v}$ decays in the Standard Model and beyond. JHEP 02, 184 (2015). https://doi.org/10.1007/JHEP02(2015)184. arXiv: 1409.4557 [hep-ph]

128. W. Altmannshofer, A.J. Buras, D.M. Straub, M. Wick, New strategies for New Physics search in $B \rightarrow K^{*} \nu \bar{\nu}, B \rightarrow K \nu \bar{v}$ and $B \rightarrow X_{s} v \bar{v}$ decays. JHEP 04, 022 (2009). https://doi.org/10. 1088/1126-6708/2009/04/022. arXiv:0902.0160 [hep-ph]

129. Belle Collaboration, J. Grygier et al., Search for $\boldsymbol{B} \rightarrow \boldsymbol{h} v \overline{\boldsymbol{v}}$ decays with semileptonic tagging at Belle. Phys. Rev. D 96(9), 091101 (2017). https://doi.org/10.1103/PhysRevD.96.091101. arXiv:1702.03224 [hep-ex]. (Addendum: Phys. Rev. D 97, 099902 (2018))

130. Particle Data Group Collaboration, M. Tanabashi et al., Review of Particle Physics. Phys. Rev. D 98(3), 030001 (2018). https:// doi.org/10.1103/PhysRevD.98.030001

131. J.M. Arnold, B. Fornal, M.B. Wise, Phenomenology of scalar leptoquarks. Phys. Rev. D 88, 035009 (2013). https://doi.org/10. 1103/PhysRevD.88.035009. arXiv:1304.6119 [hep-ph]

132. P. Bandyopadhyay, R. Mandal, Vacuum stability in an extended standard model with a leptoquark. Phys. Rev. D 95(3), 035007 (2017). https://doi.org/10.1103/PhysRevD.95.035007. arXiv:1609.03561 [hep-ph]

133. O. Popov, G.A. White, One Leptoquark to unify them? Neutrino masses and unification in the light of $(g-2)_{\mu}, R_{D^{(*)}}$ and $R_{K}$ anomalies. Nucl. Phys. B 923, 324-338 (2017). https://doi.org/ 10.1016/j.nuclphysb.2017.08.007. arXiv:1611.04566 [hep-ph]

134. C. Wetterich, M. Yamada, Gauge hierarchy problem in asymptotically safe gravity-the resurgence mechanism. Phys. Lett. B 770, 268-271 (2017). https://doi.org/10.1016/j.physletb.2017.04.049. arXiv:1612.03069 [hep-th]

135. J.M. Pawlowski, M. Reichert, C. Wetterich, M. Yamada, Higgs scalar potential in asymptotically safe quantum gravity. Phys. Rev. D 99(8), 086010 (2019). https://doi.org/10.1103/PhysRevD.99. 086010. arXiv:1811.11706 [hep-th]

136. F. Staub, SARAH 4: a tool for (not only SUSY) model builders. Comput. Phys. Commun. 185, 1773-1790 (2014). https://doi.org/ 10.1016/j.cpc.2014.02.018. arXiv:1309.7223 [hep-ph]

137. K. Babu, Renormalization group analysis of the KobayashiMaskawa matrix. Z. Phys. C 35, 69 (1987). https://doi.org/10. 1007/BF01561056

138. K. Sasaki, Renormalization Group Equations for the KobayashiMaskawa Matrix. Z. Phys. C 32, 149-152 (1986). https://doi.org/ 10.1007/BF01441364

139. V.D. Barger, M. Berger, P. Ohmann, Universal evolution of CKM matrix elements. Phys. Rev. D 47, 2038-2045 (1993). https://doi. org/10.1103/PhysRevD.47.2038. arXiv:hep-ph/9210260

140. P. Kielanowski, S.J. Wysozka, J. Montes de Oca Y., Renormalization Group Equations for the CKM matrix. Phys. Rev. D 78, 116010 (2008). https://doi.org/10.1103/PhysRevD.78. 116010. arXiv:0810.2097 [hep-th] 